\title{
ON THE UNIQUENESS AND NUMERICAL APPROXIMATION OF SOLUTIONS TO CERTAIN PARABOLIC QUASI-VARIATIONAL INEQUALITIES *
}

\author{
MICHAEL HINTERMÜLLER ${ }^{\dagger}$ AND CARLOS N. RAUTENBERG ${ }^{\ddagger}$
}

\begin{abstract}
A class of abstract nonlinear evolution quasi-variational inequality (QVI) problems in function space is considered. The abstract framework developed in this paper includes constraint sets of obstacle and gradient type. The paper address the existence, uniqueness and approximation of solutions when the constraint set mapping is of a special form. Uniqueness is addressed through contractive behavior of a nonlinear mapping whose fixed points are solutions to the QVI. An axiomatic semi-discrete approximation scheme is developed, which is proven to be convergent and which is numerically implemented. The paper ends by a report on numerical tests for several nonlinear constraints of gradient-type.
\end{abstract}

Key words. Quasi-variational inequality, gradient constraints, obstacle problem, semismooth Newton method.

AMS subject classifications. 47J20, 49J40, 49M15, 65J15, 65K10

1. Introduction. The Signorini Problem is a linear elastostatic problem that was introduced by Fichera in [11 and it is the first variational inequality (VI) in the scientific literature. However, the term "variational inequality" was coined by Lions and Stampacchia in their seminal work [28, where the first abstract approach establishing existence, uniqueness and approximation techniques for VIs was developed. In the aforementioned paper, not only the extension of the famous Lax-Milgram result is established (leading to the renowned Lions-Stampacchia Theorem) but also semi-coercive and parabolic problems are dealt with.

In [7, Brézis introduced the concept of a pseudo-monotone operator and successfully applied it to parabolic VIs. In the same monograph, Brézis considered the use of an infinitesimal generator of a $C_{0}$-semigroup to describe the "time derivative" of the problem. This approach provided access to monotonicity techniques, known for elliptic problems, to treat evolution VIs. In this setting, the entire theory is built on the relationship between the closed, convex, non-empty constraint set and the $C_{0^{-}}$ semigroup that gives rise to the unbounded operator related to the time derivative.

Quasi-variational inequalites (QVIs) were introduced by Bensoussan and Lions in [5] and 26] to formulate impulse control problems and applications to several phenomena. This type of problems arises in diverse areas of applied sciences that include game theory, solid mechanics, elastoplasticity and superconductivity. For an account of models and their analytical properties we refer, e.g., to 6, 9, 14, 27, 31, 33, 23 and the monographs [3, 22, 34, as well as the references therein.

The scientific literature is rather scarce when it comes to QVIs in function space. More specifically, in function space most of the literature concerning QVIs is devoted

* This work was supported by the Austrian Ministry of Science and Education and the Austrian Science Fund FWF through START-Project Y305 "Interfaces and Free Boundaries", the German Research Fundation DFG Research Center MATHEON through Projects C28, C31 and Projects SE5 and SE15 funded by the Einstein Center for Mathematics Berlin (ECMath) and DFG Priority Program SPP 1253 through subproject "Elliptic Mathematical Programs with Equilibrium Constraints (MPECs) in Function Space: Optimality Conditions and Numerical Realization".

${ }^{\dagger}$ Department of Mathematics, Humboldt-University of Berlin, Unter den Linden 6, 10099 Berlin, Germany. (hint@math.hu-berlin.de).

${ }^{\ddagger}$ Department of Mathematics, Humboldt-University of Berlin, Unter den Linden 6, 10099 Berlin, Germany. (carlos.rautenberg@math.hu-berlin.de). 
to two types of problems: the obstacle- and the gradient-type constrained problem. While the first one studies problems where the state (or solution) to the QVI has to satisfy pointwise constraints on a given subset of the domain, the second type of problem determines a pointwise bound on the norm of the gradient of such a solution. The different constraint structure in these two problems developed into two completely different mathematical approaches: obstacle-type problems have been attacked by means of increasing monotonicity techniques (fixed point type results for increasingly monotonic mappings such as Birkhoff, Tartar or Kolodner fixed point theorems) for the solution mapping with respect to the obstacle (see [4, 12, 13, 24, 38); problems with gradient-type constraints have been treated by means of compactness results. This was done either by the direct combination of continuity of the solution mapping with respect to the upper bound on the gradient constraint in composition with some completely continuous operator such as in [23, 16] or by fine properties of compactness in Lesbesgue-Bochner spaces as in 35, 2. An alternative approach to gradient constrained problems is based on generalized equations, with the QVI problem becoming a particular case; see [18, 21. For finite dimensional problems, recently a technique based on generalized KKT conditions was pursued in 10. The latter approach, however, seems unlikely to be applicable in infinite dimensions for the problem class under investigation in our paper.

Although existence of solutions to QVIs in function space may be obtained by a variety of fixed point type theorems (e.g., Schauder in [23, Leray-Schauder in 29] and see [3] for diverse applications for monotonically increasing mappings), uniqueness results for QVI problems seem to be more difficult to obtain. In the obstacle-type QVI, uniqueness under assumptions which are rather straightforward to verify was obtained by Laetsch in 24] and a contraction type result was obtained by Hanouzet and Joly in [13. For the gradient-type problem a result of uniqueness based on contraction was given in [16] together with the numerical implementation of a newly developed solution algorithm. The difficulty in obtaining uniqueness results for QVIs comes from a variety of sources: for example, using Schauder's fixed point theorem, uniqueness results usually require differentiability (see [20]) of the mapping under investigation (differentiability properties, however, are usually difficult, if not impossible, to obtain for the mappings involved in QVIs) and in the case of some gradient constrained problems (see for example [33]) it is known that the physical system does not posses a unique steady state or fixed point.

In [17, the pseudomonotonicity and $C_{0}$-semigroup approach of Brézis was applied to parabolic QVIs in combination with approximation methods for infinitesimal generators (similar to the analytical forms of the Trotter-Kato theorem). The result is an approximation theorem that is suitable for numerical implementation when the constraint set mapping is of gradient-type and the set is causal, i.e., the solution to the QVI at time $t, u(t)$, can be obtained as a solution to a QVI where the constraint set depends only on the set $\{v: v=u(\tau)$ for $0 \leq \tau \leq t\}$. However, this approach cannot be applied to non-causal sets. The present paper addresses such non-causal problems.

In this paper we study an abstract version of a parabolic QVI which contains both, the obstacle- and gradient-type constrained problems, respectively. Within a unified framework we provide existence and uniqueness results based on a contraction type property. The result can be considered as an extension of the one obtained in 16] for elliptic QVIs. We also provide a proof of convergence in function space of a semi-discrete scheme that is suitable for numerical implementation. The result is 
based on monotone operator theory, the previous contraction result and semismooth Newton methods for solving the associated subproblems. We end this paper by providing numerical tests involving the Laplace and the $p$-Laplace (for $p=3$ ) operator, respectively, and for gradient constrained problems.

The rest of the paper is organized as follows. In $\$ 2$ we state the class of QVI problems under consideration, how this framework includes both problems with obstacleand gradient-type constraints, and how QVIs arise in the modeling of many physical phenomena. Since solutions to QVIs can be considered as fixed points of a certain mapping $S$, in Theorem 3.2 of $\$ 3$ we show that the mapping under investigation is contractive given small data or given a small Lipschitz constant of the nonlinear mapping associated with the bound in the constraint. Also in \$3 a class of examples with obstacle and gradient constraints is addressed and it is shown how the previous contraction result applies to these cases. In $\$ 4$ we state an abstract framework to deal with approximating problems to the QVI of interest. We show in detail that the scheme includes the semi-discrete version of the parabolic QVI under investigation with either the obstacle- or gradient-type constraint. Theorem 4.5 in 44 states how the mapping $S$ (whose fixed point are solutions to the QVI) and its discretized version are related through the weak topology on the state space. Theorem 4.5 together with Proposition 4.1 are used in Corollary 3.3 to show that the algorithm used in the numerical implementation is convergent. Numerical tests are carried out in $\$ 5$ and a discussion of the results, as well as, an outlook on future research directions are given in 86 .

1.1. Notation. Throughout this paper, for a Banach space $X$ its norm is written as $|\cdot|_{X}$ and for $f \in X^{\prime}$ (the topological dual of $X$ ) we denote $f(x):=(f, x)_{X^{\prime}, X}$ for $x \in X$, unless $X=\mathscr{V}$ with $\mathscr{V}$ the state space selected for the problems. In the latter case, for the sake of brevity and simplicity, we write $(f, v)$ for $f \in \mathscr{V}^{\prime}$ and $v \in \mathscr{V}$ as duality pairing. If $H$ is a Hilbert space and we identify it with its dual $H^{*}$, then we denote the duality pairing as $\langle f, x\rangle_{H}$ for $f \in H^{*}$ and $x \in H$.

The natural and real numbers are denoted by $\mathbb{N}$ and $\mathbb{R}$, respectively, and by $\mathbb{R}^{+}$ we denote the set of positive real numbers and $\mathbb{R}_{0}^{+}=\mathbb{R}^{+} \cup\{0\}$.

For $v_{0} \in X$ and $R>0$ we use $B_{R}\left(v_{0}\right):=\left\{v \in X:\left|v-v_{0}\right|_{X}<R\right\}$ (or $B_{R}\left(v_{0} ; X\right)$ ) and its closure in $X$ by $\bar{B}_{R}\left(v_{0}\right)$ (or $\bar{B}_{R}\left(v_{0} ; X\right)$ ). We denote the strong convergence of a sequence $\left\{u_{n}\right\} \subset X$ to $u \in X$ by $u_{n} \rightarrow u$. Weak convergence is written as $u_{n} \rightarrow u$. The Lebesgue measure of a measurable set $\Omega$ is denoted as $|\Omega|$, and we say that a property holds "a.e. in $\Omega$ ", if it is true in $\Omega$ except for a subset $\Omega_{0} \subset \Omega$ such that $\left|\Omega_{0}\right|=0$. For a real-valued function $v$, we define $v^{+}=\max (0, v)$ in the pointwise sense, that is, $v^{+}=v$ if $v$ is nonnegative and zero otherwise.

Let $\mathrm{I}=(0, T)$, with $0<T \leq \infty$, and $X$ be a Banach space. A function $f: \mathrm{I} \rightarrow X$ is Bochner measurable, if there is a sequence $\left\{f_{n}\right\}$ of simple $X$-valued functions such that $\lim _{n \rightarrow \infty} f_{n}(t)=f(t)$ a.e. in I (see [15]). We denote by $L^{p}(\mathrm{I} ; X)$ the (LebesgueBochner) space of Bochner measurable $X$-valued mappings with domain I such that $\int_{\mathrm{I}}|f(t)|_{X}^{p} \mathrm{~d} t<\infty$ and the integral is taken in the sense of Lebesgue.

Let $\Omega \subset \mathbb{R}^{N}$, with $N \geq 2$, be a bounded and open domain. We write $L^{p}(\Omega)$ (or $L^{p}(\Omega ; \mathbb{R})$ ) for the usual Lebesgue spaces of real-valued functions, and $L_{\nu}^{\infty}(\Omega):=$ $\left\{v \in L^{\infty}(\Omega): v(\mathbf{x}) \geq \nu>0\right.$ a.e. $\left.\mathbf{x} \in \Omega\right\}$. We denote by $W_{0}^{1, p}(\Omega)$ for $1<p<\infty$ the Sobolev space of weakly differentiable functions in $L^{p}(\Omega)$ with zero value at the boundary $\partial \Omega$ (in the sense of the trace), whose weak derivatives also belong to $L^{p}(\Omega)$ (see [1] for a definition of the Sobolev space). It is endowed with the norm $|v|_{W_{0}^{1, p}}=$ $\left(\int_{\Omega}|\nabla v(\mathbf{x})|^{p} \mathrm{~d} \mathbf{x}\right)^{1 / p}$. 
Since we will deal with convergence of closed and convex subsets of reflexive Banach spaces, we make use of Mosco convergence (see [30, 34]).

Definition 1.1 (Mosco Convergence). Let $K$ and $K_{n}$, for each $n \in \mathbb{N}$, be non-empty, closed and convex subsets of $X$, a reflexive Banach space. We say that the sequence $\left\{K_{n}\right\}$ converges to $K$ in the sense of Mosco as $n \rightarrow \infty$ if:

i. $\forall v \in K, \exists v_{n} \in K_{n}: v_{n} \rightarrow v$ in $X$.

ii. If $v_{n} \in K_{n}$ and $v_{n} \rightarrow v$ in $X$ with $n \in \mathbb{N}^{\prime} \subset \mathbb{N}$, then $v \in K$.

2. Problem Formulation. Let $\mathscr{V}$ be a reflexive separable Banach space and $\mathscr{H}$ be a separable Hilbert space so that $\left(\mathscr{V}, \mathscr{H}, \mathscr{V}^{\prime}\right)$ is a Gelfand triple, i.e., the embedding $\mathscr{V} \hookrightarrow \mathscr{H}$ is dense and continuous, $\mathscr{H}$ is identified with its dual $\mathscr{H}^{\prime}$ and hence the embedding $\mathscr{H}^{\prime}=\mathscr{H} \hookrightarrow \mathscr{V}^{\prime}$ is also continuous (see [8]). For $f \in \mathscr{V}^{\prime}$ and $v \in \mathscr{V}$ the duality pairing $(f, v)$ is supposed to be the continuous extension of $\langle\cdot, \cdot\rangle_{\mathscr{H}}$ on $\mathscr{H} \times \mathscr{V}$; so that there is a sequence $\left\{h_{n}\right\} \subset \mathscr{H}$ for which $(f, v)_{\mathscr{V}}=\lim _{n \rightarrow \infty}\left\langle h_{n}, v\right\rangle_{\mathscr{H}}$ uniformly on bounded sets of $\mathscr{V}$.

Unless stated otherwise, $\mathscr{V}=L^{p}(\mathrm{I} ; V)$ and $\mathscr{H}=L^{2}(\mathrm{I} ; H)$, where $p \geq 2, \mathrm{I}=(0, T)$ for $0<T<\infty$ and $\left(V, H, V^{\prime}\right)$ a Gelfand triple with $V$ a separable reflexive Banach space and $H$ a separable Hilbert space. Also, if $T=\infty$, then we take $\mathscr{V}=L^{2}(\mathrm{I} ; V)$ and $\mathscr{H}=L^{2}(\mathrm{I} ; H)$. In this case, since $\mathrm{I}=(0, T)$ is $\sigma$-finite, there is a concrete characterization of the dual of $\mathscr{V}$ as $\mathscr{V}^{\prime}=L^{p^{\prime}}\left(\mathrm{I} ; V^{\prime}\right)$ by the Phillips Theorem (see 37]. or [15]).

We assume that the (usually nonlinear) map $\mathcal{A}: \mathscr{V} \rightarrow \mathscr{V}^{\prime}$ is

H1. uniformly monotone, i.e., there are constants $c>0$ and $r>1$ such that,

$$
(\mathcal{A}(u)-\mathcal{A}(v), u-v) \geq c|u-v|_{\mathscr{V}}^{r}, \quad \text { for all } u, v \in \mathscr{V}
$$

H2. hemicontinuous, i.e., the real-valued function $\zeta \mapsto(\mathcal{A}(u+\zeta v), w)$ is continuous for $\zeta \in[0,1]$ for all $u, v, w \in \mathscr{V}$;

H3. bounded, i.e., it maps bounded sets in $\mathscr{V}$ into bounded sets in $\mathscr{V}^{\prime}$.

Since $\mathscr{V}$ is assumed to be reflexive, then $\mathbf{H} \mathbf{1}$ together with $\mathbf{H 2}$ imply that $\mathcal{A}$ is pseudomonotone (see [37]), i.e., if $u_{n} \rightarrow u$ and $\varlimsup_{n \rightarrow \infty}\left(\mathcal{A}\left(u_{n}\right), u_{n}-u\right)_{\mathscr{V}} \leq 0$, then $(\mathcal{A}(u), u-v)_{\mathscr{V}} \leq \underline{\lim }_{n \rightarrow \infty}\left(\mathcal{A}\left(u_{n}\right), u_{n}-v\right)$, for all $v \in \mathscr{V}$, and demicontinuous, i.e., if $u_{n} \rightarrow u$ in $\mathscr{V}$, then $\mathcal{A}\left(u_{n}\right) \rightarrow \mathcal{A}(u)$ in the weak-star topology and hence $\mathcal{A}\left(u_{n}\right) \rightarrow \mathcal{A}(u)$ in $\mathscr{V}^{\prime}$ (due to the reflexivity of $\mathscr{V}$ ).

In order to introduce some form of "time derivative", we make use of $C_{0}$-semigroup theory. To the best of our knowledge, this approach was pioneered (for variational problems associated with monotone operators) by Brézis (see [7]). For that matter, we assume in the following that $-L$ be the infinitesimal generator of a $C_{0}$-semigroup $S(\tau)$ in $\mathscr{V}, \mathscr{H}$ and $\mathscr{V}^{\prime}$ with domains $\mathscr{D}(L ; \mathscr{V}), \mathscr{D}(L ; \mathscr{H})$ and $\mathscr{D}\left(L ; \mathscr{V}^{\prime}\right)$, respectively (see 32 for the concept of a $C_{0}$-semigroup). Additionally, we assume that $S(\tau)$ is a $C_{0}$-semigroup of contractions in $\mathscr{H}$. Summarising, we suppose that for $\tau \in[0, \infty)$, $S(\tau)$ belongs $\mathscr{L}(\mathscr{V}), \mathscr{L}(\mathscr{H})$ and $\mathscr{L}\left(\mathscr{V}^{\prime}\right)$, such that $|S(\tau)|_{\mathscr{L}(\mathscr{H})} \leq 1$ for all $\tau \geq 0$ and in addition

a. $S(0)=I=$ id, the identity operator in $\mathscr{V}, \mathscr{H}$ and $\mathscr{V}^{\prime}$;

b. $S(\tau+\rho)=S(\tau) S(\rho)$ for all $\tau, \rho \geq 0$;

c. $\forall v \in \mathscr{V}, \lim _{\tau \downarrow 0} S(\tau) v=v$ in $\mathscr{V}$ and the same holds true when $\mathscr{V}$ is exchanged for $\mathscr{H}$ and $\mathscr{V}^{\prime}$.

The domain $\mathscr{D}(L ; \mathscr{V})$ is defined as

$$
\mathscr{D}(L ; \mathscr{V}):=\left\{v \in \mathscr{V}: \lim _{\tau \downarrow 0} \frac{S(\tau) v-v}{\tau} \text { exists in } \mathscr{V}\right\}
$$


where $\mathscr{D}(L ; \mathscr{H})$ and $\mathscr{D}\left(L ; \mathscr{V}^{\prime}\right)$ are defined similarly. The perhaps most common example is stated next

EXAmPle 2.1. Let $\mathscr{V}=L^{p}(\mathrm{I} ; X)$, for $\mathrm{I}=(0, T)$ with $0<T \leq \infty$, with $X$ a Banach space. For $f \in \mathscr{V}$, let $S(\tau)$ be defined by

$$
(S(\tau) f)(t)= \begin{cases}f(t-\tau), & \tau<t<T \\ 0, & \text { otherwise }\end{cases}
$$

Clearly $S(0)=I=\mathrm{id}, S\left(\tau_{1}+\tau_{2}\right)=S\left(\tau_{1}\right) S\left(\tau_{2}\right)$ and $\lim _{\tau \downarrow 0} S(\tau) f=f$ in $\mathscr{V}$. Hence $S(\tau)$ is a $C_{0}$-semigroup over $\mathscr{V}$. Moreover, $S(\tau)$ is a $C_{0}$-semigroup of contractions (since $|S(\tau) f|_{\mathscr{V}} \leq|f|_{\mathscr{V}}$ ) which is not uniformly continuous. Its domain is determined by

$$
\mathscr{D}(L ; \mathscr{V})=\left\{v \in \mathscr{V}: v \text { is absolutely continuous, } v^{\prime} \in \mathscr{V} \text { and } v(0)=0\right\}
$$

where $v^{\prime}$ is the pointwise strong derivative; for a proof see [17] or [25].

Suppose that $\mathscr{C}$ is a closed and convex subset of $\mathscr{V}, 0 \in \mathscr{C}$, and that there exist $r>0$ such that $\bar{B}_{r}(0 ; \mathscr{V}) \subset \mathscr{C}$. Consider the (usually nonlinear) map $\Phi: \mathscr{C} \rightarrow \mathscr{E}_{\nu} \subset \mathscr{E}$ where

$$
\begin{aligned}
& \mathscr{E}:=L^{\infty}\left(\mathrm{I} ; L^{\infty}(\Omega)\right)^{M}, \quad \mathscr{E}_{\nu}:=L^{\infty}\left(\mathrm{I} ; L^{\infty}(\Omega)\right)^{M-1} \times L^{\infty}\left(\mathrm{I} ; L_{\nu}^{\infty}(\Omega)\right) \quad \text { and } \\
& L_{\nu}^{\infty}(\Omega):=\left\{\varphi \in L^{\infty}(\Omega): \varphi(x) \geq \nu>0 \text { a.e. in } \Omega\right\},
\end{aligned}
$$

where $M \in \mathbb{N}$. If $\varphi=\left\{\varphi_{m}\right\}_{m=1}^{M} \in \mathscr{E}$, then we define $|\varphi|_{\mathscr{E}}:=\sum_{m=1}^{M}\left|\varphi_{m}\right|_{L^{\infty}\left(\mathrm{I} ; L^{\infty}(\Omega)\right)}$ as its norm. It should be noted that for $\varphi \in \mathscr{E}_{\mathscr{V}}$ we have $|\varphi|_{\mathscr{E}} \geq \nu>0$.

Also, consider the set-valued map $\mathscr{K}: \mathscr{E} \rightarrow 2^{\mathscr{V}}$ such that the map $\mathscr{K}(\Phi(\cdot))$ : $\mathscr{C} \rightarrow 2^{\mathscr{V}}$ satisfies that $\mathscr{K}(\Phi(v))$ is a closed and convex subset of $\mathscr{V}$ and $0 \in \mathscr{K}(\Phi(v))$, for each $v \in \mathscr{C}$. Let $f \in \mathscr{V}^{\prime}$ and $\mathcal{A}: \mathscr{V} \rightarrow \mathscr{V}^{\prime}$, then we define the problem $(\mathrm{P})$ as the following parabolic QVI.

\section{Problem (P)}

Find $u \in \mathscr{K}(\Phi(u)) \cap \mathscr{D}\left(L ; \mathscr{V}^{\prime}\right):(L u+\mathcal{A}(u)-f, v-u) \geq 0, \quad \forall v \in \mathscr{K}(\Phi(u)) . \quad(\mathrm{P})$

The space $\mathscr{V}$ is considered to be a Banach space of mappings of the type $f: \mathrm{I} \rightarrow V$ where $\mathrm{I}=(0, T)$ with $0<T \leq \infty$ and $V$ is a separable reflexive Banach space. Then a general form of $\mathscr{K}(\cdot)$ is given by

$$
\mathscr{K}(\Phi(v))=\{w \in \mathscr{V}: w(t) \in \mathbf{K}(\Phi(v), t) \text { a.e. } t \in \mathrm{I}\}
$$

where $\mathbf{K}: \mathscr{E} \times \mathrm{I} \rightarrow 2^{V}$ and, for each $w \in \mathscr{V}$ and $t \in \mathrm{I}, \mathbf{K}(\Phi(w), t)$ is a closed and convex subset of $V$ with $0 \in \mathbf{K}(\Phi(w), t)$.

The following problem will be called the weak form of problem $(\mathrm{P})$.

\section{Problem (wP)}

Find $u \in \mathscr{K}(\Phi(u)):(L v+\mathcal{A}(u)-f, v-u)_{\mathscr{V}} \geq 0, \quad \forall v \in \mathscr{K}(\Phi(u)) \cap \mathscr{D}\left(L ; \mathscr{V}^{\prime}\right) .(\mathrm{wP})$

If $u$ is a solution to $(\mathrm{PP})$, then it is also a solution $(\mathrm{wP}$ and if $u$ solves $\sqrt{\mathrm{wP}}$ and $u \in \mathscr{D}\left(L ; \mathscr{V}^{\prime}\right)$ then it also solves $(\mathrm{P})$ (see $[25]$ ). 
2.1. Popular Constraint Sets. The two most important forms for the constraint set $\mathbf{K}$ are the following ones.

Gradient-type. Let $G \in \mathscr{L}(V, W)$, a bounded linear operator with domain in $V$ and image in $W$, a Banach space of functions over some domain $\Omega \subset \mathbb{R}^{N}$ and range in $\mathbb{R}^{l}$ be given. In this case, $\Phi: \mathscr{V} \rightarrow \mathscr{E}_{\nu}$ with $\mathscr{E}_{\nu}=L^{\infty}\left(\mathrm{I} ; L_{\nu}^{\infty}(\Omega)\right)$ and

$$
\mathbf{K}_{\text {grad }}(v, t):=\left\{y \in V:|(G y)(\mathbf{x})|_{\mathbb{R}^{l}} \leq(\Phi(v)(t))(\mathbf{x}) \text { a.e. in } \Omega\right\} .
$$

Obstacle-type. Let $K \in \mathscr{L}(V, X)$, where $X$ is a Banach space of functions with domain in $\Omega$ and range in $\mathbb{R}$. Consider in this case $\Phi: \mathscr{V} \rightarrow \mathscr{E}_{\nu}$ with $\mathscr{E}_{\nu}=$ $L^{\infty}\left(\mathrm{I} ; L^{\infty}(\Omega)\right) \times L^{\infty}\left(\mathrm{I} ; L_{\nu}^{\infty}(\Omega)\right)$, such that $\Phi(v)=\left(\Phi_{1}(v), \Phi_{2}(v)\right)$, with $\left(\Phi_{1}(v)(t)\right)(\mathbf{x}) \leq$ $\left(\Phi_{2}(v)(t)\right)(\mathbf{x})$ a.e. for $t \in \mathrm{I}$ and $\mathbf{x} \in \Omega$ and with

$$
\mathbf{K}_{o b s}(v, t):=\left\{y \in V:\left(\Phi_{1}(v)(t)\right)(\mathbf{x}) \leq(K y)(\mathbf{x}) \leq\left(\Phi_{2}(v)(t)\right)(\mathbf{x}) \text { a.e. in } \Omega\right\} .
$$

The most common operators for the previous two types of constraint sets are given by $G=\nabla$ and $K=I=$ id. Hence, the condition $\Phi(\mathscr{C}) \subset \mathscr{E}_{\nu} \subset \mathscr{E}$ determines that $\nu \leq \Phi(v)$ a.e. in the gradient constrained case, and $\nu \leq \Phi_{2}(v)$ a.e. for $\Phi=\left(\Phi_{1}, \Phi_{2}\right)$ (with $\Phi_{1} \leq 0 \leq \Phi_{2}$ a.e.) in the obstacle-type constraint. This implies that we are ruling out the possibility of zero gradients and the possibility of obstacles in contact, i.e., $\Phi_{1}(x)=u(x)=\Phi_{2}(x)$ on a set of nonzero measure. Both of these situations, although perhaps not critical with respect to the proof of existence of solutions, create difficulties in the numerical approximation approach and the uniqueness of solutions to QVIs under consideration.

2.2. Practical Applications. Several practical applications of parabolic QVIs of the type considered here are discussed next.

2.2.1. The magnetization of superconductors. The magnetization of typeII superconductors has been studied by means of Bean's critical-state model. Prigozhin (in [33]) has shown that Bean's critical state model is equivalent to a QVI with gradient constraints. In the case of a stationary model with longitudinal geometry $(\Omega$ is a domain in $\mathbb{R}^{2}$ ), the main unknown $h_{z}$ is the $z$-component of the magnetic field (see [35] or 23] for the elliptic case). In this case, the constraint set is determined as

$$
\mathbf{K}(v):=\left\{y \in W_{0}^{1, p}(\Omega):|(\nabla y)(\mathbf{x})|_{\mathbb{R}^{N}} \leq j_{c}\left(\left|v+h_{e}\right|\right) \text { a.e. in } \Omega\right\},
$$

where $p \geq 2, h_{e}$ is related to the density of external currents and $j_{c}$ is an operator associated with the critical current density value. Defining $u=h-h_{e}$, the pertinent QVI problem is given by: Find $u \in \mathscr{K}(u)$ such that

$$
\left(u^{\prime}-\frac{\rho_{0}}{\mu} \Delta_{p}(u)-f, v-u\right) \geq 0 \quad \forall v \in \mathscr{K}(u),
$$

with $\mathscr{K}(v):=\{w \in \mathscr{V}: w(t) \in \mathbf{K}(v)\}, \rho_{0}>0$ a constant related to the scalar resistivity, $\mu>0, \Delta_{p}$ is the $p$-Laplacian, i.e.,

$$
\left(-\Delta_{p}(w), v\right):=\int_{\Omega}|\nabla w|^{p-2} \nabla w \cdot \nabla v \mathrm{~d} \mathbf{x}
$$

with $u^{\prime} \in \mathscr{V}^{\prime}$ and $u(0)=u_{0} \in W_{0}^{1, p}(\Omega)$, and where $f$ is also related to external currents. 
2.2.2. Elastic membrane with compliant obstacles. Let $\Omega$ be some domain in $\mathbb{R}^{N}$ with $N=1$ or $N=2$. Consider an elastic homogeneous membrane, whose displacement is denoted by $u$ and which is zero at $t=0$, that occupies the entire domain $\Omega$ and that has zero displacement on the boundary, i.e., $\left.u\right|_{\partial \Omega}=0$. Suppose that the membrane is loaded by the uniformly distributed force $f$ and that there are two obstacles $\Phi_{1} \leq 0 \leq \Phi_{2}$ a.e. such that $\Phi_{1} \leq u \leq \Phi_{2}$ a.e. on $\Omega$ constraining the deflection of the membrane. In this case, consider $\mathscr{V}=L^{2}(\mathrm{I} ; V)$ and $\mathscr{H}=$ $L^{2}(\mathrm{I} ; H)$, where $\left(V, H, V^{\prime}\right) \equiv\left(H_{0}^{1}(\Omega), L^{2}(\Omega), H^{-1}(\Omega)\right)$. Also, we have $K \equiv i d \in$ $\mathscr{L}\left(H_{0}^{1}(\Omega), H_{0}^{1}(\Omega)\right.$ ) (where $K$ is as in 2.2 ) leading to

$$
\mathbf{K}=\left\{y \in H_{0}^{1}(\Omega): \Phi_{1}(\mathbf{x}) \leq y(\mathbf{x}) \leq \Phi_{2}(\mathbf{x}) \text { a.e. in } \Omega\right\} .
$$

Then a simplified model for the evolutionary dynamics of the problem is given by: Find $u \in \mathscr{K}:=\{v \in \mathscr{V}: v(t) \in \mathbf{K}\}$ with $u^{\prime} \in \mathscr{V}^{\prime}$ and $u(0)=0$ such that

$$
\left(u^{\prime}-\Delta(u)-f, v-u\right) \geq 0, \quad \forall v \in \mathscr{K} .
$$

The associated QVI version of the above parabolic VI modeling the dynamic obstacle problem can be considered as the problem where the obstacles $\Phi_{1}, \Phi_{2}$ are not "fixed" but rather depend on the displacement of the membrane $u$ (for example, this situation would consider that when the membrane is in contact with some obstacle, the latter suffers a force exerted by the membrane that determines its movement). In this case we also have $K \equiv i d \in \mathscr{L}\left(H_{0}^{1}(\Omega), H_{0}^{1}(\Omega)\right)$. However, for $v \in \mathscr{V}$, let $\Phi(v):=$ $\left(\Phi_{1}(v), \Phi_{2}(v)\right)$ with $\left(\Phi_{1}(v), \Phi_{2}(v)\right) \in L^{\infty}(\Omega) \times L_{\nu}^{\infty}(\Omega)$ such that $\Phi_{1}(v) \leq 0 \leq \Phi_{2}(v)$ a.e. for all $v \in \mathscr{V}$. Hence, we obtain

$$
\mathbf{K}(v):=\left\{y \in H_{0}^{1}(\Omega):\left(\Phi_{1}(v)\right)(\mathbf{x}) \leq y(\mathbf{x}) \leq\left(\Phi_{2}(v)\right)(\mathbf{x}) \text { a.e. in } \Omega\right\} .
$$

Then, the QVI problem amounts to finding $u \in \mathscr{K}(\Phi(u))$ with $u^{\prime} \in \mathscr{V}^{\prime}$ and $u(0)=0$ such that

$$
\left(u^{\prime}-\Delta(u)-f, v-u\right) \geq 0, \quad \forall v \in \mathscr{K}(\Phi(u)) .
$$

3. Conditions for $u \mapsto S(A, f, \mathscr{K}(\Phi(u)))$ to be contractive. Let $f, \mathscr{K}$ and $S(\tau)$, the $C_{0}$-semigroup that is generated by $-L$, satisfy conditions so that $S(\mathcal{A}, f, \mathscr{K})$ is well defined as the unique solution to

$$
\text { Find } u \in \mathscr{D}(L ; \mathscr{V}) \cap \mathscr{K}:(L u+\mathcal{A}(u)-f, v-u) \geq 0, \quad \forall v \in \mathscr{K} .
$$

Conditions for this to hold are for example given by $f \in \mathscr{D}\left(L ; \mathscr{V}^{\prime}\right), \mathscr{K}=\{v \in \mathscr{V}$ : $v(t) \in K$ a.e. $\}$ with $K$ some closed, convex set in $V$ with $0 \in K$ and $S(\tau)$ given by Example 2.1. (see for example 25, 7]). When $\mathscr{K}$ is not constant, we assume that each evaluation $\mathscr{K}(v)$ satisfies the previous condition. If $t \mapsto K(t)$ is not constant, and $K(t)$ is of the obstacle- or gradient-type, then regularity and growth conditions on the obstacle or gradient bounds are required in order to ensure existence and uniqueness of the solution to 3.1. (see, for example, section 5.2 in [17). In the setting of Theorem 3.2 below, for the gradient constraint case, this would require second-order in time regularity and more stringent growth conditions on the function $\phi$.

Note, however, that weaker forms of solutions could be considered in 3.1. Then analogous results to the ones developed subsequently hold true for the appropriate QVI formulation. In fact, if there is a unique solution $\tilde{S}(\mathcal{A}, f, \mathscr{K})$ to:

$$
\text { Find } u \in \mathscr{V} \cap \mathscr{K}, \partial_{t} u \in \mathscr{V}^{\prime}: \quad\left(\partial_{t} u+\mathcal{A}(u)-f, v-u\right) \geq 0, \quad \forall v \in \mathscr{K},
$$


with $u(0)=0$, where $\partial_{t} u$ denotes the weak partial derivative in time of $u$, and where $\mathscr{K}=\mathscr{K}(\Phi(v))$ for any $v \in \mathscr{C}$, then Theorem 3.2 and Corollary 3.3 also hold for $\tilde{S}$.

In this section we establish conditions for contractibility of the solution mapping $u \mapsto S(A, f, \mathscr{K}(\Phi(u)))$. We start with some preliminary results of stability and continuity of $\mathcal{A} \mapsto S(\mathcal{A}, f, \mathscr{K})$.

Proposition 3.1. Let $\mathcal{A}_{1}$ and $\mathcal{A}_{2}$ satisfy $\mathbf{H 1}$ (with $c_{1}>0, r_{1}>1$ and $c_{2}>$ $0, r_{2}>1$, respectively), $\mathbf{H} \mathbf{2}$ and $\mathbf{H 3}$, then

$$
\left|S\left(\mathcal{A}_{2}, f, \mathscr{K}\right)-S\left(\mathcal{A}_{1}, f, \mathscr{K}\right)\right|_{\mathscr{V}} \leq M\left(\delta\left(\mathcal{A}_{2}, \mathcal{A}_{1}\right)\right)^{\frac{1}{\bar{r}-1}},
$$

where $\bar{r}=\min \left(r_{1}, r_{2}\right)$, for some $M>0$, and

$$
\delta\left(\mathcal{A}_{2}, \mathcal{A}_{1}\right):=\sup _{v \in \bar{B}_{R}(0 ; \mathscr{V})}\left|\mathcal{A}_{2}(v)-\mathcal{A}_{1}(v)\right|_{\mathscr{V}^{\prime}},
$$

with $R:=\max \left(\left(|f|_{\mathscr{V}^{\prime}} / c_{1}\right)^{1 /\left(r_{1}-1\right)},\left(|f|_{\mathscr{Y}^{\prime}} / c_{2}\right)^{1 /\left(r_{2}-1\right)}\right)$.

Proof. Without loss of generality suppose that $r_{2} \leq r_{1}$. Define $u_{i}=S\left(\mathcal{A}_{i}, f, \mathscr{K}\right)$ for $i=1,2$. Since $u_{i}$ solves $(\mathrm{P})$, it also solves $(\mathrm{wP})$. Let $v=0$ in $(\mathrm{wP})$, then $\left(\mathcal{A}_{i}\left(u_{i}\right), u_{i}\right) \leq\left(f, u_{i}\right)$ and hence $\left|u_{i}\right|_{\mathscr{V}} \leq R$ (by the uniform monotonicity of $\mathcal{A}_{i}$ for $i=1,2)$. Since $u_{1}, u_{2} \in \mathscr{K}$, we have

$$
\left(L u_{1}+\mathcal{A}_{1}\left(u_{1}\right)-f, u_{2}-u_{1}\right) \geq 0 \quad \text { and } \quad\left(L u_{2}+\mathcal{A}_{2}\left(u_{2}\right)-f, u_{1}-u_{2}\right) \geq 0 .
$$

Hence, from these two inequalities, we infer

$$
\left(L\left(u_{2}-u_{1}\right), u_{2}-u_{1}\right)_{\mathscr{V}}+\left(\mathcal{A}_{2}\left(u_{2}\right)-\mathcal{A}_{2}\left(u_{1}\right), u_{2}-u_{1}\right)_{\mathscr{V}} \leq\left(\mathcal{A}_{1}\left(u_{1}\right)-\mathcal{A}_{2}\left(u_{1}\right), u_{2}-u_{1}\right)_{\mathscr{V}} .
$$

If $w \in \mathscr{D}\left(L ; \mathscr{V}^{\prime}\right) \cap \mathscr{K}$, then $(L w, w)=\lim _{\tau \downarrow 0} \frac{1}{\tau}(I-S(\tau) w, w)_{\mathscr{V}}=\lim _{\tau \downarrow 0} \frac{1}{\tau}\left(|w|_{\mathscr{H}}^{2}-\right.$ $\left.\langle S(\tau) w, w\rangle_{\mathscr{H}}\right) \geq 0$, since $S(\tau)$ is a $C_{0}$-semigroup of contractions over $\mathscr{H}$.

Then, due to the uniform monotonicity of $\mathcal{A}_{2}$, we have

$$
c_{2}\left|u_{2}-u_{1}\right|_{\mathscr{V}}^{r_{2}} \leq\left(\mathcal{A}_{2}\left(u_{2}\right)-\mathcal{A}_{2}\left(u_{1}\right), u_{2}-u_{1}\right) \leq\left(\mathcal{A}_{1}\left(u_{1}\right)-\mathcal{A}_{2}\left(u_{1}\right), u_{2}-u_{1}\right) .
$$

Since $\left|u_{i}\right|_{\mathscr{V}} \leq R$, we find $\left(\mathcal{A}_{1}\left(u_{1}\right)-\mathcal{A}_{2}\left(u_{1}\right), u_{2}-u_{1}\right) \leq \delta\left(\mathcal{A}_{2}, \mathcal{A}_{1}\right)\left|u_{2}-u_{1}\right|_{\mathscr{V}}$, which implies

$$
\left|u_{2}-u_{1}\right|_{\mathscr{V}} \leq\left(2 R / c_{2}\right)^{1 /\left(r_{2}-1\right)}\left(\delta\left(\mathcal{A}_{2}, \mathcal{A}_{1}\right)\right)^{1 /\left(r_{2}-1\right)} .
$$

We now state the main result of the paper which guarantees the contractivity of the map $u \mapsto S(A, f, \mathscr{K}(\Phi(u)))$ under certain conditions. The result can be seen as the extension of the one in [16] for elliptic QVIs to the parabolic case.

TheOrem 3.2. Let $\mathscr{V} \equiv L^{p}(\mathrm{I} ; V)$ with $\mathrm{I}=(0, T)$, where $\left(V, H, V^{\prime}\right)$ is a Gelfand triple and $1<p<\infty$ if $|\mathrm{I}|<\infty$ (and $p=2$ if $|\mathrm{I}|=\infty$ ). In addition, suppose

i. $\mathcal{A}: \mathscr{V} \rightarrow \mathscr{V}^{\prime}$ satisfies $\mathbf{H 1}$ with $\min (2, p) \geq r>1$ if $|\mathrm{I}|<\infty \quad$ (and $r=2$ if $\left.|\mathrm{I}|=\infty\right)$, H2, H3 and is homogeneous of order $\beta \geq 1$, i.e., for fixed $s>0$, we have $s^{\beta} A(v)=A(s v)$ for all $v \in V$.

ii. $f \in L^{r^{\prime}}\left(\mathrm{I} ; V^{\prime}\right) \subset L^{p^{\prime}}\left(\mathrm{I} ; V^{\prime}\right)$, such that $(f, v)=\int_{\mathrm{I}}(f(t), v(t))_{V} \mathrm{~d} t$ for all $v \in \mathscr{V} \equiv$ $L^{p}(\mathrm{I} ; V)$, where $1 / r+1 / r^{\prime}=1$ and $1 / p+1 / p^{\prime}=1$.

iii. $\mathscr{K}: \mathscr{E} \rightarrow 2^{\mathscr{V}}$, satisfies that if $\varphi \in \mathscr{E}_{\nu} \subset \mathscr{E}$, then $\alpha \mathscr{K}(\varphi)=\mathscr{K}(\alpha \varphi)$ for all $\alpha>0$. iv. $\Phi: \mathscr{C} \subset \mathscr{V} \rightarrow \mathscr{E}_{\nu} \subset \mathscr{E}$ is defined as $\Phi(u)=\Gamma(u) \phi$ with $\phi=\left\{\phi_{m}\right\}_{m=1}^{M} \in \mathscr{E} \equiv$ $L^{\infty}\left(\mathrm{I} ; L^{\infty}(\Omega)\right)^{M}$ and $\Gamma: \mathscr{C} \rightarrow \mathbb{R}$ such that there exists 
a. $\underline{\gamma}>0$ with

$$
\underline{\gamma} \leq \Gamma(u), \quad \forall u \in \bar{B}_{R}(0 ; \mathscr{V})
$$

where $R:=\left(|f|_{L^{r^{\prime}}\left(\mathrm{I} ; V^{\prime}\right)}\right)^{1 /(r-1)}$.

b. $L_{\Gamma}>0$ for which

$$
|\Gamma(v)-\Gamma(w)| \leq L_{\Gamma}|v-w|_{\mathscr{V}}, \quad \forall v, w \in \bar{B}_{R}(0 ; \mathscr{V}) .
$$

Then, the map $u \mapsto S(\mathcal{A}, f, \mathscr{K}(\Phi(u)))$ satisfies $S(\mathcal{A}, f, \mathscr{K}(\Phi(\cdot))): \bar{B}_{R}(0 ; \mathscr{V}) \rightarrow$ $\bar{B}_{R}(0 ; \mathscr{V})$ and

$$
\left|S\left(\mathcal{A}, f, \mathscr{K}\left(\Phi\left(u_{2}\right)\right)\right)-S\left(\mathcal{A}, f, \mathscr{K}\left(\Phi\left(u_{1}\right)\right)\right)\right|_{\mathscr{V}} \leq L_{S}(f)\left|u_{2}-u_{1}\right|_{\mathscr{V}},
$$

for all $u_{1}, u_{2} \in \bar{B}_{R}(0 ; \mathscr{V})$ and some $L_{S}(f)>0$ such that $\lim _{|f|_{L^{r^{\prime}\left(\mathrm{I} ; V^{\prime}\right)}} \rightarrow 0} L_{S}(f)=0$. Moreover, $L_{S}(f)=\mathcal{O}\left(L_{\Gamma}\right)$ implying $\lim _{L_{\Gamma} \rightarrow 0} L_{S}(f)=0$.

Proof. First, note that $S(\mathcal{A}, f, \mathscr{K}(\Phi(v))) \in \mathscr{D}(L ; \mathscr{V}) \cap \mathscr{K}(\Phi(v))$, for each $v \in$ $\bar{B}_{R}(0 ; \mathscr{V}) \subset \mathscr{C}$, is well defined as the unique solution to (3.1) (with $\mathscr{K}=\mathscr{K}(\Phi(v))$ ) by the initial hypotheses and the first paragraph of this section.

Let $\varphi \in \mathscr{E}_{\nu}, \varphi \in$ Range $\Phi$, and denote $\mathscr{K} \equiv \mathscr{K}(\varphi)$. Also, define $u_{i}=S\left(\mathcal{A}, f_{i}, \mathscr{K}\right)$ for $i=1,2$. Then,

$$
\left(L\left(u_{2}-u_{1}\right)+\mathcal{A}\left(u_{2}\right)-\mathcal{A}\left(u_{1}\right), u_{2}-u_{1}\right) \leq\left(f_{2}-f_{1}, u_{2}-u_{1}\right) .
$$

The uniform monotonicity of $\mathcal{A}$ and $(L w, w) \geq 0, \forall w \in \mathscr{D}\left(L ; \mathscr{V}^{\prime}\right) \cap \mathscr{K}$ imply (as in the proof of Proposition 3.1 that

$$
c\left|u_{2}-u_{1}\right|_{\mathscr{V}}^{r} \leq\left(f_{2}-f_{1}, u_{2}-u_{1}\right) .
$$

Young's inequality states $\int_{\Omega}|g v| \mathrm{d} \mathbf{x} \leq \frac{\epsilon^{r^{\prime}}}{r^{\prime}} \int_{\Omega}|g|^{r^{\prime}} \mathrm{d} \mathbf{x}+\frac{1}{r \epsilon^{r}} \int_{\Omega}|v|^{r} \mathrm{~d} \mathbf{x}$, for all $g \in L^{r^{\prime}}(\Omega)$, $v \in L^{r}(\Omega)$, and all $\epsilon>0$. Now, since $p \geq r$, we obtain by Young's and Hölder's (when $p>r)$ inequalities

$$
\begin{aligned}
c\left|u_{2}-u_{1}\right|_{\mathscr{V}}^{r} & \leq\left(f_{2}-f_{1}, u_{2}-u_{1}\right)=\int_{\mathrm{I}}\left(\left(f_{2}-f_{1}\right)(t),\left(u_{2}-u_{1}\right)(t)\right)_{V} \mathrm{~d} t \\
& \leq \frac{\epsilon^{r^{\prime}}}{r^{\prime}} \int_{\mathrm{I}}\left|\left(f_{1}-f_{2}\right)(t)\right|_{V^{\prime}}^{r^{\prime}} \mathrm{d} t+\frac{1}{r \epsilon^{r}} \int_{\mathrm{I}}\left|\left(u_{1}-u_{2}\right)(t)\right|_{V}^{r} \mathrm{~d} t \\
& \leq \frac{\epsilon^{r^{\prime}}}{r^{\prime}} \int_{\mathrm{I}}\left|\left(f_{1}-f_{2}\right)(t)\right|_{V^{\prime}}^{r^{\prime}} \mathrm{d} t+\frac{|\mathrm{I}|^{(p-r) / p}}{r \epsilon^{r}}\left(\int_{\mathrm{I}}\left|\left(u_{1}-u_{2}\right)(t)\right|_{V}^{p} \mathrm{~d} t\right)^{r / p} .
\end{aligned}
$$

Hence for a sufficiently large $\epsilon>0$

$$
\left|u_{2}-u_{1}\right|_{\mathscr{V}} \leq\left(\frac{\frac{\epsilon^{r^{\prime}}}{r^{\prime}}}{\left(c-\frac{|\mathrm{I}|(p-r) / p}{r \epsilon^{r}}\right)}\right)^{1 / r}\left(\int_{\mathrm{I}}\left|\left(f_{1}-f_{2}\right)(t)\right|_{V^{\prime}}^{r^{\prime}} \mathrm{d} t\right)^{1 / r} .
$$

In the case when $|\mathrm{I}|=\infty$ (and then $p=r=2$ by the initial hypotheses), we similarly have

$$
c\left|u_{2}-u_{1}\right|_{\mathscr{V}}^{2} \leq \frac{\epsilon^{2}}{2} \int_{\mathrm{I}}\left|\left(f_{1}-f_{2}\right)(t)\right|_{V^{\prime}}^{2} \mathrm{~d} t+\frac{1}{2 \epsilon^{2}}\left(\int_{\mathrm{I}}\left|\left(u_{1}-u_{2}\right)(t)\right|_{V}^{2} \mathrm{~d} t\right),
$$


and again for $\epsilon>0$ large enough,

$$
\left|u_{2}-u_{1}\right|_{\mathscr{V}} \leq\left(\frac{\epsilon^{2}}{\left(c-\frac{1}{2 \epsilon^{2}}\right)}\right)^{1 / 2}\left(\int_{\mathrm{I}}\left|\left(f_{1}-f_{2}\right)(t)\right|_{V^{\prime}}^{2} \mathrm{~d} t\right)^{1 / 2} .
$$

Therefore,

$$
\left|S\left(\mathcal{A}, f_{1}, \mathscr{K}\right)-S\left(\mathcal{A}, f_{2}, \mathscr{K}\right)\right|_{\mathscr{V}} \leq M_{1}\left|f_{2}-f_{1}\right|_{L^{r^{\prime}\left(\mathrm{I} ; V^{\prime}\right)}}^{r^{\prime} / r}
$$

where $M_{1}>0$ depends on $c, p, r$ and $|\mathrm{I}|$ if the latter is finite, otherwise it depends only on $c$ (given that $p=r=2$ if $|\mathrm{I}|=\infty$ ).

Suppose that $\mu>0$, then $\mu^{1-\beta} \mathcal{A}$ (for $\beta \geq 1$ ) satisfies $\mathbf{H 1}, \mathbf{H} 2$ and $\mathbf{H 3}, \mu f \in \mathscr{V}^{\prime}$ and $\mu \mathscr{K}$ is closed, convex and $0 \in \mu \mathscr{K}$. Hence, we find

$$
\begin{gathered}
S(\mathcal{A}, f, \mathscr{K})-S(\mathcal{A}, f, \mu \mathscr{K})=\left(S(\mathcal{A}, f, \mathscr{K})-S\left(\mu^{1-\beta} \mathcal{A}, \mu f, \mu \mathscr{K}\right)\right)+ \\
\left(S\left(\mu^{1-\beta} \mathcal{A}, \mu f, \mu \mathscr{K}\right)-S(\mathcal{A}, \mu f, \mu \mathscr{K})\right)+(S(\mathcal{A}, \mu f, \mu \mathscr{K})-S(\mathcal{A}, f, \mu \mathscr{K})) \\
=I+I I+I I I .
\end{gathered}
$$

(where all evaluations of the mapping $S$ are well defined).

Consider $I$. Let $u=S(\mathcal{A}, f, \mathscr{K})$, then $(L u+\mathcal{A}(u)-f, v-u) \geq 0, \forall v \in \mathscr{K}$. Since $L$ is a linear operator, and $\mathcal{A}$ is homogeneous of order $\beta \geq 1$, for $\mu>0$ we have $\left(L(\mu u)+\mu^{1-\beta} \mathcal{A}(\mu u)-\mu f, w-\mu u\right) \geq 0, \forall w \in \mu \mathscr{K}$, i.e., $\mu u=S\left(\mu^{1-\beta} \mathcal{A}, \mu f, \mu \mathscr{K}\right)$. Then,

$$
|I|_{\mathscr{V}} \leq \Theta_{I}(f)|1-\mu|,
$$

where $\Theta_{I}(f)=|S(\mathcal{A}, f, \mathscr{K})|_{\mathscr{V}}$, and as argued before (see the proof of Proposition 3.1) $|S(\mathcal{A}, f, \mathscr{K})|_{\mathscr{V}} \leq\left(|f|_{\mathscr{V}^{\prime}} / c\right)^{1 /(r-1)}$. Since $p \geq r>1$, we infer $r^{\prime} \geq p^{\prime}>1$, and hence $L^{r^{\prime}}\left(\mathrm{I} ; V^{\prime}\right) \hookrightarrow \mathscr{V}^{\prime} \equiv L^{p^{\prime}}\left(\mathrm{I} ; V^{\prime}\right)$, where the embedding is continuous. Consequently, we obtain $\lim _{|f|_{L^{r^{\prime}\left(\mathrm{I} ; V^{\prime}\right)}} \rightarrow 0} \Theta_{I}(f)=0$.

In order to find a bound on $I I$, we apply Proposition 3.1. In this case $\mathcal{A}$ and $\mu^{1-\beta} \mathcal{A}$ satisfy $\mathbf{H} \mathbf{1}$ with the same $r$ and with $c$ and $\mu^{1-\beta} c$, respectively. Then,

$$
|I I|_{\mathscr{V}} \leq \Theta_{I I}(f)\left|1-\mu^{\beta-1}\right|^{\frac{1}{r-1}}, \quad \Theta_{I I}(f):=\left(\frac{2 R}{c} \sup _{w \in \bar{B}_{R}(0 ; \mathscr{V})}|\mathcal{A}(w)|_{\mathscr{V}^{\prime}}\right)^{\frac{1}{r-1}}
$$

with $R \leq\left(\mu^{1 /(r-1)}+\mu^{\beta /(r-1)}\right)\left(|f|_{\mathscr{V}^{\prime}} / c\right)^{1 /(r-1)}$ (where $R$ is the one in Proposition 3.1). Since $\mathcal{A}$ maps bounded sets in $\mathscr{V}$ into bounded sets in $\mathscr{V}^{\prime}$ (Hypothesis H3),

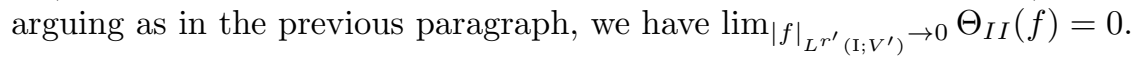

We now use 3.3 to bound $I I I$. This yields (note that $\frac{1}{r-1}=\frac{r^{\prime}}{r}$ )

$$
|I I I|_{\mathscr{V}} \leq \Theta_{I I I}(f)|1-\mu|^{\frac{1}{r-1}},
$$

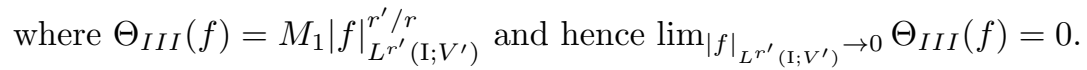

Suppose that $\mu \in(0, \bar{\mu}]$ for some fixed $\bar{\mu}>0$. Since $2 \geq r>1$ and $\beta \geq 1$, it holds that $\left|1-\mu^{\beta-1}\right|^{1 /(r-1)} \leq \delta_{1}|1-\mu|$ and $|1-\mu|^{1 /(r-1)} \leq \delta_{2}|1-\mu|$ for some $\delta_{1}>0$ and $\delta_{2}>0$ (depending only on $\bar{\mu}$ ) for all $\mu \in(0, \bar{\mu}]$. Then, from $(3.4)$, we observe that

$$
|S(\mathcal{A}, f, \mathscr{K})-S(\mathcal{A}, f, \mu \mathscr{K})|_{\mathscr{V}} \leq \Theta(f)|1-\mu|,
$$


where $\Theta(f):=\Theta_{I}(f)+\delta_{1} \Theta_{I I}(f)+\delta_{2} \Theta_{I I I}(f)$.

We have that $\mathscr{K} \equiv \mathscr{K}(\varphi)$ for some $\varphi \in \mathscr{E}_{\nu}$ and that $\mu \mathscr{K}(\varphi)=\mathscr{K}(\mu \varphi)$, and we write, for the sake of brevity, $S(\varphi):=S(\mathcal{A}, f, \mathscr{K})$ and $S(\mu \varphi):=S(\mathcal{A}, f, \mu \mathscr{K})$. Since $\varphi \in \mathscr{E}_{\nu}$, we have $|\varphi|_{\mathscr{E}} \geq \nu>0$, and hence

$$
|S(\varphi)-S(\mu \varphi)|_{\mathscr{V}} \leq \frac{\Theta(f)}{\nu}|1-\mu||\varphi|_{\mathscr{E}} \leq \frac{\Theta(f)}{\nu}|\varphi-\mu \varphi|_{\mathscr{E}} .
$$

Finally, let $\varphi=\Gamma\left(u_{2}\right) \phi$ and $\mu=\Gamma\left(u_{1}\right) / \Gamma\left(u_{2}\right)$. Since $u_{1}, u_{2} \in \mathscr{C}$, and hence, since $u \mapsto \Gamma(u)$ is Lipschitz on $\mathscr{C}$ (with Lipschitz constant $L_{\Gamma}$ by assumption), we have

$$
\left|S\left(\Gamma\left(u_{2}\right) \phi\right)-S\left(\Gamma\left(u_{1}\right) \phi\right)\right|_{\mathscr{V}} \leq \frac{\Theta(f)}{\nu}\left|\Gamma\left(u_{2}\right) \phi-\Gamma\left(u_{1}\right) \phi\right|_{\mathscr{E}} \leq \frac{\Theta(f)|\phi|_{\mathscr{E}} L_{\Gamma}}{\nu}\left|u_{2}-u_{2}\right|_{\mathscr{V}} .
$$

Therefore $u \mapsto S(\mathcal{A}, f, \mathscr{K}(\Phi(u)))$ is Lipschitz continuous and contractive for all sufficiently small $f$. Moreover, the Lipschitz constant of $S, L_{S}(f)$, is proportional to $L_{\Gamma}$.

Remark 1. It should be noted that the map $u \mapsto S(\mathcal{A}, f, \mathscr{K}(\Phi(u)))$ is nonlinear (even in the case when $\mathcal{A}$ is linear, due to the constraints) and hence the contractive behavior (and consequently the existence of a unique solution) should be expected only for small data, i.e., small $f$ in the $L^{r^{\prime}}\left(\mathrm{I} ; V^{\prime}\right)$-sense. Given the structure of the constraint mapping $v \mapsto \mathscr{K}(\Phi(v))$ required for the previous theorem, one might think that small $f$ forces the system into "inactivity", i.e., that $u=S(\mathcal{A}, f, \mathscr{K}(\Phi(u)))$ belongs to the interior of $\mathscr{K}(\Phi(u))$ and, hence, the problem being dealt with is no longer a proper QVI but satisfies $L u+\mathcal{A}(u)-f=0$. This, however, is not the case! Indeed, for any $f$, one can choose a small enough $L_{\Gamma}$ to obtain the contractive behavior of the map $S$ (and hence uniqueness).

Remark 2. The Lipschitz constant of $\Gamma, L_{\Gamma}$, controls how much $v \mapsto \Phi(v)=\Gamma(v) \phi$ changes on the ball $\bar{B}_{R}(0 ; \mathscr{V})$. If $L_{\Gamma}=0$, then the QVI problem reduces to a VI, which has a unique solution. In this sense, as $L_{\Gamma} \downarrow 0$, it is expected that the properties of (P) resemble more and more the ones of a VI. The previous theorem is evidence of such a behavior. In addition, the Lipschitz behavior of $\Gamma$ implies the same for $S$. On the other hand, differentiability properties of $S$ are in general not implied by differentiability of $\Gamma$.

The following corollary is a direct consequence of the previous result and determines a direct and natural way for approximating solutions to the QVI of interest. The proof is simply an application of Theorem 3.2 and Banach's fixed point principle.

Corollary 3.3. Suppose the hypotheses of Theorem 3.2 are satisfied and that $|f|_{L^{r^{\prime}\left(\mathrm{I} ; V^{\prime}\right)}}$ is small enough. Define

$$
T(v):=S(\mathcal{A}, f, \mathscr{K}(\Phi(v))),
$$

and consider $u_{n}=T\left(u_{n-1}\right)$ for $n \in \mathbb{N}$ and $u_{0} \in \bar{B}_{R}(0 ; \mathscr{V})$. Then, the sequence $\left\{u_{n}\right\}$ converges (at least, linearly) in the strong topology of $\mathscr{V}$ to $u^{*}$, the unique solution $u=T(u)$.

Remark. Note that even though the approach of the paper is not concentrated on existence results for QVIs, the result in Corollary 3.3 neither contains nor is contained in general existence results like the ones in [18].

Theorem 3.2 and Corollary 3.3 now allow to establish the existence and uniqueness of solutions to certain classes of parabolic QVIs, and they provide a way of approximating these. Furthermore, note that the two aforementioned results also hold for $\tilde{S}$ 
as defined in 3.2 under minor changes, provided $\tilde{S}(\mathcal{A}, f, \mathscr{K}(\Phi(w)))$ is well defined for each $w \in \overline{B_{R}}(0 ; \mathscr{V})$. In this case, we observe that there is a unique solution to $u=\tilde{S}(\mathcal{A}, f, \mathscr{K}(\Phi(u)))$, i.e., $u$ satisfies the QVI:

$u \in \mathscr{V} \cap \mathscr{K}(\Phi(u)), \quad \partial_{t} u \in \mathscr{V}^{\prime}: \quad\left(\partial_{t} u+\mathcal{A}(u)-f, v-u\right) \geq 0, \quad \forall \in \mathscr{K}(\Phi(u))$.

A class of examples for the gradient type and obstacle type constrained are given in the following.

EXAMPLE 3.1. Let $p=2, \mathscr{V}=L^{2}(\mathrm{I} ; V)$ with $\mathrm{I}=(0, T)$ and $\left(V, H, V^{\prime}\right)=$ $\left(H_{0}^{1}(\Omega), L^{2}(\Omega), H^{-1}(\Omega)\right.$. Let $\mathcal{A}: \mathscr{V} \rightarrow \mathscr{V}^{\prime}$ be the time realization of the Laplacian, i.e., $\mathcal{A}(v)(t)=A(v(t))$ with $A=-\Delta$ for all $v \in \mathscr{V}$, which satisfies $\mathbf{H 1}$ (with $r=2$ and $c=1), \mathbf{H} 2$ and $\mathbf{H 3}$ and is homogeneous of order $\beta=1$. Let $f \in L^{2}\left(\mathrm{I} ; V^{\prime}\right)$ so that $(f, v)_{\mathscr{V}}=\int_{\mathrm{I}}(f(t), v(t))_{V} \mathrm{~d} t$.

Consider $\mathscr{K}: \mathscr{E}_{\nu} \subset \mathscr{E} \rightarrow 2^{\mathscr{V}}$ where $\mathscr{E}_{\nu}=L^{\infty}\left(\mathrm{I} ; L_{\nu}^{\infty}(\Omega)\right), \mathscr{E}=L^{\infty}\left(\mathrm{I} ; L^{\infty}(\Omega)\right)$ and

$$
\mathscr{K}(\varphi)=\left\{v \in L^{2}\left(\mathrm{I} ; H_{0}^{1}(\Omega)\right):|(\nabla v(t))(x)|_{\mathbb{R}^{l}} \leq(\varphi(t))(\mathbf{x}) \text { a.e. for } t \in \mathrm{I}, \mathbf{x} \in \Omega\right\},
$$

which satisfies that $\alpha \mathscr{K}(\varphi)=\mathscr{K}(\alpha \varphi)$ for all $\alpha>0, \varphi \in \mathscr{E}_{\nu}$. Let

$$
\Gamma(u)=k|\Psi(u)|+\nu, \quad \text { with } \Psi \in \mathscr{V}^{\prime} \text { and } \forall u \in \mathscr{V},
$$

$k>0$ and $\phi \equiv 1$ such that $\Phi(u)=\Gamma(u) \phi=\Gamma(u)$. In this case, we observe that for all $u \in L^{2}\left(\mathrm{I} ; H_{0}^{1}(\Omega)\right)$, the set $\mathscr{K}(\Phi(u))$ is a closed and non-empty subset of $L^{2}\left(\mathrm{I} ; H_{0}^{1}(\Omega)\right)$ and contains 0 . Then, by Theorem 3.2. the mapping $u \mapsto S(\mathcal{A}, f, \mathscr{K}(\Phi(u)))$ is Lipschitz continuous and contractive on some ball provided that $|f|_{L^{2}\left(\mathrm{I} ; V^{\prime}\right)}($ or $k>0)$ is small enough.

EXAMPLE 3.2. Consider again $p=2, \mathscr{V}=L^{2}(\mathrm{I} ; V)$ with $\mathrm{I}=(0, T)$ and $\left(V, H, V^{\prime}\right)=\left(H_{0}^{1}(\Omega), L^{2}(\Omega), H^{-1}(\Omega)\right.$. Let $\mathcal{A}: \mathscr{V} \rightarrow \mathscr{V}^{\prime}$ be $\mathcal{A}(v)(t)=A(v(t))$ with $A=$ $-\Delta$ as in the previous example and let $f \in L^{2}\left(\mathrm{I} ; V^{\prime}\right)$ with $(f, v)_{\mathscr{V}}=\int_{\mathrm{I}}(f(t), v(t))_{V} \mathrm{~d} t$.

Determine $\mathscr{K}: \mathscr{E}_{\nu} \subset \mathscr{E} \rightarrow 2^{\mathscr{V}}$ where $\mathscr{E}_{\nu}=L^{\infty}\left(\mathrm{I} ; L^{\infty}(\Omega)\right) \times L^{\infty}\left(\mathrm{I} ; L_{\nu}^{\infty}(\Omega)\right)$, $\mathscr{E}=L^{\infty}\left(\mathrm{I} ; L^{\infty}(\Omega)\right) \times L^{\infty}\left(\mathrm{I} ; L^{\infty}(\Omega)\right)$ and

$$
\mathscr{K}\left(\varphi_{1}, \varphi_{2}\right)=\left\{v \in \mathscr{V}:\left(\varphi_{1}(t)\right)(\mathbf{x}) \leq(v(t))(\mathbf{x}) \leq\left(\varphi_{2}(t)\right)(\mathbf{x}) \text { a.e. for } t \in \mathrm{I}, \mathbf{x} \in \Omega\right\},
$$

which satisfies that $\alpha \mathscr{K}(\varphi)=\mathscr{K}(\alpha \varphi)$ for all $\alpha>0, \varphi=\left(\varphi_{1}, \varphi_{2}\right) \in \mathscr{E}_{\nu}$. Consider $\Phi(\cdot)$ defined as

$$
\Phi(v)=\Gamma(v)\left(-\left|\psi_{2}\right|,\left|\psi_{1}\right|+\epsilon\right),
$$

with $\psi_{i} \in L^{\infty}\left(\mathrm{I} ; L^{\infty}(\Omega)\right), \epsilon>0$ and

$$
\Gamma(v)=k|\Psi(u)|+\delta, \quad \text { with } \Psi \in \mathscr{V}^{\prime} \text { and } \forall u \in \mathscr{V},
$$

$k>0$ and $\epsilon \delta \geq \nu>0$. Hence, we have $\Phi: \mathscr{V} \rightarrow \mathscr{E}_{\nu} \subset \mathscr{E}$. Also, by Theorem 3.2, the mapping $u \mapsto S(\mathcal{A}, f, \mathscr{K}(\Phi(u)))$ is Lipschitz continuous and contractive on some ball provided that $|f|_{L^{2}\left(\mathrm{I} ; V^{\prime}\right)}$ (or $k>0$ ) is small enough.

3.1. Approximations in a General Setting. The previous result may also be useful when the constraint map $\Phi$ has a different structure compared to the one required in Theorem 3.2. In fact, in some cases it is possible to construct a sequence of approximating problems for which the theory still applies. Indeed, we sketch such an approximation procedure in what follows. For this purpose we confine ourselves to the obstacle type example with $\Phi(v):=\left(0, \Phi_{2}(v)\right)$ and where $\Phi_{2}: H_{0}^{1}(\Omega) \rightarrow(\nu,+\infty)$ 
is Lipschitz continuous with constant $L_{\Phi}$ and a forcing term $f(t)=f \in L^{2}(\Omega)$, for all $t \in[0, T]$.

Let $I_{n}=\left[t_{n-1}, t_{n}\right)$ for $n=1,2, \ldots, N, t_{n}:=n T / N$, and consider the sequence of maps $\left\{\Lambda_{n}\right\}$ with $\Lambda_{n}: L^{2}\left(I_{n} ; H_{0}^{1}(\Omega)\right) \rightarrow(\nu,+\infty)$ defined by $\Lambda_{n}(v):=$ $\frac{1}{\left|I_{n}\right|} \int_{I_{n}} \Phi_{2}(v(t)) \mathrm{d} t$. Note that each $\Lambda_{n}$ is Lipschitz continuous with constant $L_{\Phi}$. Also, if $w \in L^{2}\left((0, T) ; H_{0}^{1}(\Omega)\right)$, then standard integration results yield that $\frac{1}{h} \int_{s}^{s+h} \Phi_{2}(v(t)) \mathrm{d} t \rightarrow$ $\Phi_{2}(v(s))$ as $h \downarrow 0$ for almost all $s \in(0, T)$. Since $|f|_{L^{2}\left(I_{n} ; L^{2}(\Omega)\right)}=\left|I_{n}\right|^{1 / 2}|f|_{L^{2}(\Omega)}$, then for sufficiently large $N,|f|_{L^{2}\left(I_{n} ; L^{2}(\Omega)\right)}$ gets arbitrarily small. Then, Theorem 3.2 and Corollary 3.3 can be applied to $T(v):=S\left(\mathcal{A}, f, \mathscr{K}\left(\Lambda_{n}(v)\right)\right.$ ) (or $\tilde{T}(v):=$ $\left.S\left(\mathcal{A}, f, \mathscr{K}\left(\Lambda_{n}(v)\right)\right)\right)$ on $\mathscr{V}:=L^{2}\left(I_{n} ; H_{0}^{1}(\Omega)\right)$, for $n=1$. Provided the maps $S$ and $\tilde{S}$ are also uniquely defined for non-zero initial conditions (under certain assumptions on $u_{0}$ ), the same procedure can be repeated for $n>1$, provided that $|f|_{L^{2}(\Omega)}$ and $\left|u_{0}\right|_{H_{0}^{1}(\Omega)}$ are sufficiently small. In this way one approximates the solution to the original problem.

Note, however, that the well-posedness of the maps $S$ (and $\tilde{S}$ ) with non-zero boundary conditions $u(0)=u_{0} \neq 0$ may be a challenging problem in its own right (see [21], section 5.4 in [17] and [25]), which requires additional studies as $N \rightarrow \infty$.

In the gradient constrained case of magnetization of superconductors, the upper bound of the gradient constraint operator $j_{c}$ is in general a superposition (or Nemytskii) operator such that the scheme above is not directly applicable. However, an approximation of the magnetization problem for $p=2$ can be obtained when $j_{c}: H_{0}^{1}(\Omega) \rightarrow L^{2}(\Omega)$ is replaced by $\hat{j}_{c}(v)=j_{c}(S v)$ with $S v=\frac{1}{|\Omega|} \int v(x) \mathrm{d} x$. In this case, the above procedure can be applied with the obvious changes.

4. A Semi-Discrete Scheme. Let $\left\{X_{n}\right\}$ be a sequence of Banach spaces related to a Banach space $X$ by the following extension and projection operators.

Assumption 1. For $n \in \mathbb{N}$, there are $P_{n} \in \mathscr{L}\left(X, X_{n}\right)$ and $E_{n} \in \mathscr{L}\left(X_{n}, X\right)$ such that

A1. For all $n \in \mathbb{N}$, we have $\left|P_{n}\right|_{\mathscr{L}\left(X, X_{n}\right)} \leq 1$ and $\left|E_{n}\right|_{\mathscr{L}\left(X_{n}, X\right)} \leq 1$.

A2. $\left|E_{n} P_{n} v-v\right|_{\mathscr{V}} \rightarrow 0$ as $n \rightarrow \infty$ for all $v \in X$.

A3. $P_{n} E_{n}$ is the identity operator in $X_{n}$.

TheOREM 4.1. Let $X$ and $X_{n}$ for $n \in \mathbb{N}_{0}$ be Banach spaces related by projection and extension operators $P_{n}$ and $E_{n}$ that satisfy $\mathbf{A} \mathbf{1}$ and A3. Let $T: X \rightarrow X$ and $T_{n}: X_{n} \rightarrow X_{n}$ be a sequence of contractive operators such that

$$
\left|T_{n}(x)-T_{n}(y)\right|_{X_{n}} \leq \eta_{n}|x-y|_{X_{n}} \quad \text { with } \quad \bar{\eta}:=\sup _{n \in \mathbb{N}} \eta_{n}<1 .
$$

Consider the sequence of operators $\hat{T}_{n}: X \rightarrow X$ defined as $\hat{T}_{n}(x)=E_{n} T_{n}\left(P_{n} x\right)$ for each $x \in X$.

If there exists $x_{0} \in X$ with $\left|T_{n}\left(P_{n} x_{0}\right)\right|_{X_{n}} \leq K$ for all $n \in \mathbb{N}$ and $\hat{T}_{n}$ satisfies

$$
\lim _{n \rightarrow \infty} \hat{T}_{n}\left(x_{n}\right)=T(x) \text {, in } X \text { if } x_{n} \rightarrow x \text { in } X,
$$

then the sequence of fixed points

$$
y_{n}=T_{n}\left(y_{n}\right),
$$

satisfies that $\left\{E_{n} y_{n}\right\}$ converges strongly to the unique fixed point of $T$.

Proof. First we prove that $T$ has a unique fixed point. By the definition of $\hat{T}_{n}$, and the fact that the norms of $E_{n} \in \mathscr{L}\left(X_{n}, X\right)$ and $P_{n} \in \mathscr{L}\left(X, X_{n}\right)$ are uniformly 
bounded by 1 , we have that

$$
\left|\hat{T}_{n}(x)-\hat{T}_{n}(y)\right|_{X} \leq\left|T_{n}\left(P_{n} x\right)-T_{n}\left(P_{n} y\right)\right|_{X_{n}} \leq \eta_{n}\left|P_{n} x-P_{n} y\right|_{X_{n}} \leq \bar{\eta}|x-y|_{X} .
$$

Then we infer from 4.2

$$
|T(x)-T(y)|_{X}=\lim _{n \rightarrow \infty}\left|\hat{T}_{n}(x)-\hat{T}_{n}(y)\right|_{X} \leq \bar{\eta}|x-y|_{X},
$$

i.e., $T: X \rightarrow X$ is contractive and hence has a unique fixed point.

Consider the sequence of fixed points $y_{n}=T_{n}\left(y_{n}\right)$. Since $P_{n} E_{n}$ is the identity, we have that $E_{n} y_{n}=E_{n} T_{n}\left(P_{n} E_{n} y_{n}\right)$. Then defining $\hat{y}_{n}:=E_{n} y_{n}$, we have that $\hat{y}_{n}=\hat{T}_{n}\left(\hat{y}_{n}\right)$. The sequence $\left\{\hat{y}_{n}\right\}$ is uniformly bounded. Indeed, we have

$$
\left|\hat{y}_{n}\right|_{X}-\left|\hat{T}_{n}\left(x_{0}\right)\right|_{X} \leq\left|\hat{T}_{n}\left(\hat{y}_{n}\right)-\hat{T}_{n}\left(x_{0}\right)\right|_{X} \leq \bar{\eta}\left|\hat{y}_{n}-x_{0}\right|_{X} \leq \bar{\eta}\left|\hat{y}_{n}\right|_{X}+\bar{\eta}\left|x_{0}\right|_{X},
$$

and hence

$$
(1-\bar{\eta})\left|\hat{y}_{n}\right|_{X} \leq\left|\hat{T}_{n}\left(x_{0}\right)\right|_{X}+\bar{\eta}\left|x_{0}\right|_{X} \leq\left|T_{n}\left(P_{n} x_{0}\right)\right|_{X_{n}}+\bar{\eta}\left|x_{0}\right|_{X} \leq K+\bar{\eta}\left|x_{0}\right|_{X} .
$$

Therefore, $\hat{y}_{n_{i}} \rightarrow y^{*}$ in $X$ and $\hat{T}_{n_{i}}\left(\hat{y}_{n_{i}}\right) \rightarrow T\left(y^{*}\right)$ by 4.2$)$. Since $\hat{y}_{n_{i}}=\hat{T}_{n_{i}}\left(\hat{y}_{n_{i}}\right)$, we obtain $y^{*}=T\left(y^{*}\right)$.

Suppose that there is a subsequence of $\hat{y}_{n}=\hat{T}_{n}\left(\hat{y}_{n}\right)$ that does not converge to $y^{*}$. Then there exists a sequence $\left\{\hat{y}_{n_{j}}\right\}$ such that $\hat{y}_{n_{j}}=\hat{T}_{n_{j}}\left(\hat{y}_{n_{j}}\right)$ and $\epsilon>0$ for which $\left|y^{*}-\hat{y}_{n_{j}}\right|_{X} \geq \epsilon>0$ for $j \in \mathbb{N}$. However, the argument at the beginning of the proof also applies to $\left\{\hat{y}_{n_{j}}\right\}$. Thus, there is a subsequence that converges to some fixed point $\bar{y}$ of the map $T$. As there is only one fixed point for $T$, we have $\bar{y}=y^{*}$. Consequently, all subsequences converge to $y^{*}$.

We consider now a semi-discretization scheme that makes the previous extension of Banach's fixed point principle useful in the study of parabolic QVIs. The abstract framework of this section is suitable for numerical methods computing approximate solutions to $(\mathrm{P})$.

Let $\mathscr{V}=L^{p}(\mathrm{I} ; V)$ (with $\left.p \geq 2\right)$ where $\mathrm{I}=(0, T)$ with $0<T<\infty$ and $\mathscr{V}_{n}=V^{n}:=$ $V \times V \times \cdots \times V(n$ copies of $V)$ with norm $|w|_{\mathscr{V}_{n}}=\left(h \sum_{m=1}^{n}\left|w^{m}\right|_{V}^{p}\right)^{1 / p}, h=\frac{T}{n}$, and where $w=\left\{w^{m}\right\}_{m=1}^{n} \in \mathscr{V}_{n}$. We assume that $\left(V, H, V^{\prime}\right)$ is a Gelfand triple and hence $\left(\mathscr{V}, \mathscr{H}, \mathscr{V}^{\prime}\right)$ and $\left(\mathscr{V}_{n}, \mathscr{H}_{n}, \mathscr{V}_{n}^{\prime}\right)$ are as well, with $\mathscr{H}=L^{2}(\mathrm{I} ; H)$ and $\mathscr{H}_{n}=H^{n}$. Then, consider $P_{n} \in \mathscr{L}\left(\mathscr{V}, \mathscr{V}_{n}\right)$ and $E_{n} \in \mathscr{L}\left(\mathscr{V}_{n}, \mathscr{V}\right)$ defined as

$$
P_{n} v:=\left\{\frac{1}{h} \int_{\mathrm{I}_{m}} v(t) \mathrm{d} t\right\}_{m=1}^{n}, \quad\left(E_{n} w\right)(t):=\sum_{m=1}^{n} w^{m} \chi_{\mathrm{I}_{m}}(t),
$$

where $v \in \mathscr{V}, w=\left\{w^{m}\right\}_{m=1}^{n} \in \mathscr{V}_{n}$ and $\mathrm{I}_{m}=((m-1) h, m h)$ for $m=1, \ldots, n$ (we also extend the latter to $m \in \mathbb{Z}$ ). We refer to $P_{n}$ and $E_{n}$ as the "projection" and "extension" operators, respectively.

Proposition 4.2. Let $P_{n}: \mathscr{V} \rightarrow \mathscr{V}_{n}$ and $E_{n}: \mathscr{V}_{n} \rightarrow \mathscr{V}$ be as defined in (4.3), then $\mathbf{A} \mathbf{1}, \mathbf{A} \mathbf{2}$ and $\mathbf{A} \mathbf{3}$ of Assumption 1 are satisfied.

Proof. It follows from the definition of $E_{n}$ and $P_{n}$ that $\mathbf{A} \mathbf{3}$ is satisfied. In order to prove $\mathbf{A} \mathbf{1}$, observe that from the definition of $E_{n}$ and the norm $|\cdot|_{\mathscr{V}_{n}}$ that $\left|E_{n} w\right|_{\mathscr{V}}=|w|_{\mathscr{V}_{n}}$ for $w \in \mathscr{V}_{n}$ and by Hölder's inequality we obtain

$$
\begin{aligned}
\left|P_{n} v\right|_{\mathscr{V}_{n}}^{p} & =h \sum_{m=1}^{n}\left|\frac{1}{h} \int_{\mathrm{I}_{m}} v(t) \mathrm{d} t\right|_{V}^{p} \leq h^{1-p} \sum_{m=1}^{n}\left(\int_{\mathrm{I}_{m}}|v(t)|_{V} \mathrm{~d} t\right)^{p} \\
& \leq \sum_{m=1}^{n} \int_{\mathrm{I}_{m}}|v(t)|_{V}^{p} \mathrm{~d} t=|v|_{\mathscr{V}}^{p} .
\end{aligned}
$$


Hence, $\left|E_{n}\right|_{\mathscr{L}\left(\mathscr{V}_{n}, \mathscr{V}\right)},\left|P_{n}\right|_{\mathscr{L}\left(\mathscr{V}, \mathscr{V}_{n}\right)} \leq 1$ and $\mathbf{A} 1$ holds. Now, we consider $\mathbf{A} 2$ and suppose that $v \in \mathscr{V}$ is of the form $v=a \chi_{\left[t_{\alpha}, t_{\beta}\right)}$ with $a \in V, 0 \leq t_{\alpha}<t_{\beta}$ and $\left[t_{\alpha}, t_{\beta}\right) \in \mathrm{I}$. Then it is elementary to check that $E_{n} P_{n} v \rightarrow v$ as $n \rightarrow \infty$ in $\mathscr{V}$. Since $E_{n} P_{n}$ is linear, then it also holds for step functions $v=\sum_{k=1}^{N} a_{k} \chi_{\left[t_{\alpha_{k}}, t_{\beta_{k}}\right)}$ with $a_{k} \in V$. Since step functions are dense in $\mathscr{V}=L^{p}(\mathrm{I} ; V)$, given $v \in \mathscr{V}$ there is a step function $v_{\epsilon}$ such that $\left|v-v_{\epsilon}\right| \leq \frac{\epsilon}{3}$. Let $n \geq N(\epsilon)$ so that $\left|E_{n} P_{n} v_{\epsilon}-v_{\epsilon}\right|_{\mathscr{V}} \leq \frac{\epsilon}{3}$. Then, we have

$$
\left|E_{n} P_{n} v-v\right|_{\mathscr{V}} \leq\left|E_{n} P_{n}\left(v-v_{\epsilon}\right)\right|_{\mathscr{V}}+\left|E_{n} P_{n} v_{\epsilon}-v_{\epsilon}\right|_{\mathscr{V}}+\left|v_{\epsilon}-v\right|_{\mathscr{V}} \leq \epsilon,
$$

given that $\left|E_{n} P_{n}\right|_{\mathscr{L}(\mathscr{V}, \mathscr{V})} \leq\left|E_{n}\right|_{\mathscr{L}\left(\mathscr{V}_{n}, \mathscr{V}\right)}\left|P_{n}\right|_{\mathscr{L}\left(\mathscr{V}, \mathscr{V}_{n}\right)} \leq 1$. Since $\epsilon>0$ was arbitrary, the assertion is proven and A2 holds. Hence, Assumption 1 holds true for our semidiscrete scheme. $\square$

Also, it is useful to note that from the definition of $P_{n}$ and $E_{n}$, we observe that the restriction of the adjoints $P_{n}^{\prime}\left(\right.$ to $\mathscr{V}_{n}$ ) and $E_{n}^{\prime}$ (to $\mathscr{V}$ ) are given by $\left.P_{n}^{\prime}\right|_{\mathscr{V}_{n}}=E_{n}$ and $\left.E_{n}^{\prime}\right|_{\mathscr{V}}=P_{n}$.

The semi-discrete problem approximating $(\mathrm{P})$ is given as follows.

Problem $\left(\mathrm{P}^{n}\right)$ :

Find $u \in \mathscr{K}_{n}\left(\Phi_{n}(u)\right):\left(L_{n} u+\mathcal{A}_{n}(u)-f_{n}, v-u\right)_{\mathscr{V}_{n}^{\prime}, \mathscr{V}_{n}} \geq 0, \quad \forall v \in \mathscr{K}_{n}\left(\Phi_{n}(u)\right),\left(\mathrm{P}^{n}\right)$

where $\left\{\mathscr{K}_{n}, \Phi_{n}, L_{n}, \mathcal{A}_{n}, f_{n}\right\}$ approximate their counterparts $\{\mathscr{K}, \Phi, L, \mathcal{A}, f\}$ in $(\mathrm{P})$ as described in the following paragraphs. We assume throughout this section that the conditions for the solution mapping $u \mapsto S(\mathcal{A}, f, \mathscr{K}(\Phi(u)))$ to be contractive from Theorem 3.2 are satisfied and conditions for $S(\mathcal{A}, f, \mathscr{K}(\Phi(v))) \in \mathscr{D}(L ; \mathscr{V}) \subset \mathscr{D}\left(L ; \mathscr{V}^{\prime}\right)$ also hold. Further conditions on $(\overline{\mathrm{P}})$ and $\sqrt{\left.\mathrm{P}^{n}\right)}$ are stated next.

Assumption 2. The following statements are assumed to hold true.

B1. The operator $L$ is the infinitesimal generator of the semigroup of translations on $\mathscr{V}$ and $\mathscr{V}^{\prime}$ (and of contractions on $\mathscr{H}$ ) defined in Example 2.1. Therefore,

$$
\mathscr{D}(L ; X)=\left\{v \in X: v \text { is absolutely continuous, } v^{\prime} \in X \text { and } v(0)=0\right\},
$$

where $X$ is $\mathscr{V}, \mathscr{H}$ or $\mathscr{V}^{\prime}$. The approximated sequence $\left\{L_{n}\right\}$ is defined as $L_{n}=$ $\frac{I-F(1 / n)}{1 / n}$, where $F(1 / n) w=\left\{0, w_{1}, w_{2}, \ldots, w_{n-1}\right\} \in \mathscr{V}_{n}$ for $w=\left\{w_{i}\right\}_{i=1}^{n} \in$ $\mathscr{V}_{n}$, i.e., $L_{n} w=\left\{\left(L_{n} w\right)_{i}\right\}_{i=1}^{n}$ with

$$
\left(L_{n} w\right)_{i}= \begin{cases}\frac{w_{1}}{1 / n}, & i=1 \\ \frac{w_{i}-w_{i-1}}{1 / n}, & 2 \leq i \leq n\end{cases}
$$

B2. $f \in \mathscr{D}(L ; \mathscr{V}) \subset \mathscr{D}\left(L ; \mathscr{V}^{\prime}\right)$ and $f_{n}=P_{n} f$.

B3. $\mathcal{A}$ is the time realization of a linear uniformly monotone operator $A$ in $V$, i.e., $\mathcal{A}(y)(t)=A(y(t))$ for $t \in \mathrm{I}$ where $A: V \rightarrow V^{\prime}$ satisfies $\boldsymbol{H 1}$, H2 and $\boldsymbol{H}$ 3. $\mathcal{A}_{n}: \mathscr{V}_{n} \rightarrow \mathscr{V}_{n}^{\prime}$ is defined as $\mathcal{A}_{n}(w)=\left\{A\left(w_{i}\right)\right\}_{i=1}^{n}$ where $w=\left\{w_{i}\right\}_{i=1}^{n} \in \mathscr{V}_{n}$. In this sense, $\mathcal{A}_{n} \equiv \mathcal{A}$.

B4. Suppose that $\phi \in\left(L^{\infty}(\Omega)\right)^{M-1} \times L_{\nu}^{\infty}(\Omega)$ so that $\phi(t)=\phi$ for all $t \in \mathrm{I}$ satisfies $\phi \in \mathscr{E}_{\nu} \subset \mathscr{E}$ with $\mathscr{E}=L^{\infty}\left(\mathrm{I} ; L^{\infty}(\Omega)\right)^{M}$, and $\mathscr{E}_{\nu}=L^{\infty}\left(\mathrm{I} ; L^{\infty}(\Omega)\right)^{M-1} \times$ $L^{\infty}\left(\mathrm{I} ; L_{\nu}^{\infty}(\Omega)\right)$. Then, we define

$$
\Phi(v)=\Gamma(v) \phi \text { and } \Phi_{n}(v)=\Gamma_{n}(v) \phi \text { for all } v \in \mathscr{V} .
$$

Consider $g \in \mathscr{V} \subset \mathscr{V}^{\prime}, \Gamma: \mathscr{V} \rightarrow \mathbb{R}$ and $\Gamma_{n}: \mathscr{V}_{n} \rightarrow \mathbb{R}$ defined as

$$
\Gamma(v)=\left|\int_{0}^{T}(g(t), v(t))_{V^{\prime}, V} \mathrm{~d} t\right|+\underline{\gamma} \quad \Gamma_{n}(w)=\left|\int_{0}^{T}\left(g(t),\left(E_{n} w\right)(t)\right)_{V^{\prime}, V} \mathrm{~d} t\right|+\underline{\gamma},
$$


with $\gamma>0$.

B5. The set-valued mappings $\mathscr{K}(\cdot)$ and $\mathscr{K}_{n}(\cdot)$ are defined as

$$
\begin{aligned}
& \mathscr{K}(\Phi(y))=\{w \in \mathscr{V}: w(t) \in \mathbf{K}(\Phi(y)) \text { a.e. } t \in \mathrm{I}\}, \\
& \mathscr{K}_{n}\left(\Phi_{n}(z)\right)=\left\{\left\{w^{m}\right\}_{m=1}^{n} \in \mathscr{V}_{n}: w^{m} \in \mathbf{K}\left(\Phi_{n}(z)\right) \text { for } m=1, \ldots, n\right\},
\end{aligned}
$$

where $y \in \mathscr{V}$ and $z_{n} \in \mathscr{V}_{n}$. We assume the following type of convergence

(i) If $v_{n} \in \mathscr{K}_{n}\left(\Phi_{n}\left(P_{n} w_{n}\right)\right), w_{n} \rightarrow w$ in $\mathscr{V}$ and $E_{n} v_{n} \rightarrow v$ in $\mathscr{V}$ for $n \in \mathbb{N}^{\prime} \subset$ $\mathbb{N}$, then $v \in \mathscr{K}(\Phi(w))$

(ii) If $w_{n} \rightarrow w$ in $\mathscr{V}$ for $n \in \mathbb{N}^{\prime} \subset \mathbb{N}$ and $v \in \mathscr{K}(\Phi(w))$, then there exists a sequence $\left\{\eta_{n}\right\} \subset \mathbb{R}^{+}$such $\lim _{n \rightarrow \infty} \eta_{n}=1$ and $\eta_{n} P_{n} v \in \mathscr{K}_{n}\left(\Phi_{n}\left(P_{n} w_{n}\right)\right)$ for $n \in \mathbb{N}^{\prime} \subset \mathbb{N}$.

Conditions B1 and B2 in Assumption 2 determine that we approximate the time derivative " $L$ " by a forward difference and invoke a more regular forcing term $f$ (when compared to the existence proof, but the additional regularity is needed for the approximation results) and its approximate $f_{n}$. These assumptions are appropriate for the kind of convergence needed in Theorem 4.5. If $v \in \mathscr{D}\left(L ; \mathscr{V}^{\prime}\right) \cap \mathscr{V}$ then $\lim _{n \rightarrow \infty} P_{n}^{\prime} L_{n} P_{n} v=L v$ in $\mathscr{V}^{\prime}$ (see Proposition 1 in [17]) and $E_{n} f_{n}=E_{n} P_{n} f \rightarrow f$ by A2, Assumption 1 . Condition B3 is clearly satisfied by $\mathcal{A}$ being the time realization of $A=-\Delta$ where $\Delta$ is the Laplacian, i.e., it is satisfied for the operator that arises in most applications. Assumptions B4 and B5 state the general form for the mappings $\Phi$ and $\Phi_{n}$ as well as the type of convergence needed for $\mathscr{K}_{n}$ towards $\mathscr{K}$. In particular, B5 is analogous to Mosco convergence of sets but written here in a form, which is more suitable for our approximation scheme. In the following paragraphs we study the implications of $\mathbf{B} \mathbf{4}$ and we show that the gradient-type and obstacle-type problems satisfy B5, respectively.

We start by considering the relationship between $\Gamma$ (the nonlinear functional in Theorem 3.2 and $\Gamma_{n}$ (the counterpart of $\Gamma$ in the approximate problem). By invoking B4 above, we assume that $\Gamma$ and $\Gamma_{n}$ satisfy the conditions necessary for Theorem 3.2 to hold true. Hence, the solution mapping of the original problem and its semidiscretized version are Lipschitz continuous and become contractive for small enough $g$ in the sense of $\mathscr{V}$. The following result relates the weak convergence in $\mathscr{V}$ and the functionals $\Gamma$ and $\Gamma_{n}$.

Proposition 4.3. Let $v_{n} \rightarrow v$ in $\mathscr{V}$. Then $\Gamma_{n}\left(P_{n} v_{n}\right) \rightarrow \Gamma(v)$.

Proof. Denote $(g, w)=\int_{0}^{T}(g(t), w(t)) \mathrm{d} t$ for $w \in \mathscr{V}$. Hence, since $\left.P_{n}^{\prime}\right|_{\mathscr{V}_{n}}=E_{n}$, $\left.E_{n}^{\prime}\right|_{\mathscr{V}}=P_{n}$ and $g \in \mathscr{V}$ we have that

$$
\left(g, E_{n} P_{n} w\right)=\left(P_{n} g, P_{n} w\right)_{\mathscr{V}_{n}^{\prime}, \mathscr{V}_{n}}=\left(E_{n} P_{n} g, w\right) .
$$

But $E_{n} P_{n}$ converges strongly to the identity by $\mathbf{A 2}$ in Assumption 11. Thus, $E_{n} P_{n} g \rightarrow$ $g$ in $\mathscr{V}$ as $n \rightarrow \infty$. Then, $\left(g, E_{n} P_{n} v_{n}\right)=\left(E_{n} P_{n} g, v_{n}\right) \rightarrow(g, v)$ and hence $\Gamma_{n}\left(P_{n} v_{n}\right) \rightarrow$ $\Gamma(v)$ follows.

In the case of the gradient constraint we have that $\mathscr{K}_{n}\left(\Phi_{n}(v)\right) \in 2^{\mathscr{V}_{n}}$ for $v \in \mathscr{V}_{n}$ and $\mathscr{K}(\Phi(z))$ for $z \in \mathscr{V}$ are given by

$$
\begin{aligned}
& \mathscr{K}(\Phi(z))=\left\{w \in \mathscr{V}:|\nabla w(t)|_{\mathbb{R}^{l}} \leq \Gamma(z) \phi \text { a.e. on } \Omega, t \in \mathrm{I}\right\} . \\
& \mathscr{K}_{n}\left(\Phi_{n}(v)\right)=\left\{\left\{w^{m}\right\}_{m=1}^{n} \in \mathscr{V}_{n}:\left|\nabla w^{m}\right|_{\mathbb{R}^{l}} \leq \Gamma_{n}(v) \phi \text { a.e. on } \Omega, \text { for } 1 \leq m \leq n\right\},
\end{aligned}
$$

and in the case of the obstacle-type problem by

$$
\begin{aligned}
& \mathscr{K}(\Phi(z))=\left\{w \in \mathscr{V}: \Gamma(z) \phi_{1} \leq w(t) \leq \Gamma(z) \phi_{2} \text { a.e. on } \Omega, t \in \mathrm{I}\right\} . \\
& \mathscr{K}_{n}\left(\Phi_{n}(v)\right)=\left\{\left\{w^{m}\right\}_{m=1}^{n} \in \mathscr{V}_{n}: \Gamma_{n}(v) \phi_{1} \leq w^{m} \leq \Gamma_{n}(v) \phi_{2} \text { a.e. on } \Omega, \text { for } 1 \leq m \leq n\right\}
\end{aligned}
$$


where "( $\mathbf{x})$ " is suppressed for the sake of clarity and brevity.

The following proposition shows that for the gradient-type and obstacle-type problems, the assumptions B5(i) and B5(ii) hold for the scheme already described above.

Proposition 4.4. Let $\mathscr{K}_{n}\left(\Phi_{n}(\cdot)\right): \mathscr{V}_{n} \rightarrow 2^{\mathscr{V}_{n}}$ and $\mathscr{K}(\Phi(\cdot)): \mathscr{V} \rightarrow 2^{\mathscr{V}}$ be as in (4.5) or 4.6). Then, B5(i) and B5(ii) hold.

Proof. Consider B5(i) for the gradient constrained case. Clearly, if $v_{n} \in \mathscr{K}_{n}\left(\Phi_{n}\left(P_{n} w_{n}\right)\right)$, then $E_{n} v_{n} \in \mathscr{K}\left(\Phi\left(E_{n} P_{n} w_{n}\right)\right)$. Since $w_{n} \rightarrow w$ in $\mathscr{V}$, then by Proposition 4.3 . $\Phi\left(E_{n} P_{n} w_{n}\right)=\Phi_{n}\left(P_{n} w_{n}\right) \rightarrow \Phi(w)$ in $\mathscr{E}=L^{\infty}\left(\mathrm{I} ; L^{\infty}(\Omega)\right)$. This implies that $\mathscr{K}\left(\Phi\left(E_{n} P_{n} w_{n}\right)\right) \rightarrow \mathscr{K}(\Phi(w))$ in the sense of Mosco (see [17, 36]) and hence that $v \in \mathscr{K}(\Phi(w))$.

For the case of the obstacle-type problem, we have that

$$
\Gamma\left(E_{n} P_{n} w_{n}\right) \phi_{1}(\mathbf{x}) \leq E_{n} v_{n}(t)(\mathbf{x}) \leq \Gamma\left(E_{n} P_{n} w_{n}\right) \phi_{2}(\mathbf{x}),
$$

a.e. for $\mathbf{x} \in \Omega, t \in$ I. Since $E_{n} v_{n} \rightarrow v$ in $\mathscr{V}$, by Mazur's Lemma there exists a convex combination $\tilde{v}_{n}=\sum_{i=1}^{N(n)} \lambda_{i}(n) E_{i} v_{i}$ such $\tilde{v}_{n} \rightarrow v$ in $\mathscr{V}$. Then the above inequality implies that $\Gamma\left(E_{n} P_{n} w_{n}\right) \phi_{1}(\mathbf{x}) \leq \tilde{v}_{n}(t)(\mathbf{x}) \leq \Gamma\left(E_{n} P_{n} w_{n}\right) \phi_{2}(\mathbf{x})$. Since $\Gamma\left(E_{n} P_{n} w_{n}\right) \rightarrow$ $\Gamma(w)$ by Proposition 4.3 , we have $\Gamma(w) \phi_{1}(\mathbf{x}) \leq v(t)(\mathbf{x}) \leq \Gamma(w) \phi_{2}(\mathbf{x})$ (since strong convergence in $\mathscr{V}$ implies a.e. pointwise convergence (along a subsequence) in the strong topology of $V$, for $t \in \mathrm{I}$, and in turn pointwise convergence in $\Omega$ along another subsequence). Hence $v \in \mathscr{K}(\Phi(w))$ also for the obstacle-type constraint, and B5(i) holds.

We turn our attention to the gradient constrained case. Since $w_{n} \rightarrow v$, then due to the definition of $\Phi$, we have that $\Phi_{n}\left(P_{n} w_{n}\right)=\Phi\left(E_{n} P_{n} w_{n}\right) \rightarrow \Phi(w)$ in $\mathscr{E}=$ $L^{\infty}\left(\mathrm{I} ; L^{\infty}(\Omega)\right.$ ) (and actually in $L^{\infty}(\Omega)$ since $\Phi(v)=\Gamma(v) \phi$ with $\phi \in L^{\infty}(\Omega)$ and similarly for $\left.\Phi_{n}\right)$ and also $\Phi\left(E_{n} P_{n} w_{n}\right), \Phi(w) \geq \nu>0$ for $n \in \mathbb{N}^{\prime} \subset \mathbb{N}$. Then, it is possible to prove (see [16]) that there is a sequence $\eta_{n} \uparrow 1$ such $\eta_{n} \Phi(w) \leq \Phi\left(E_{n} P_{n} w_{n}\right)$ for $n \in \mathbb{N}^{\prime} \subset \mathbb{N}$. Since $v \in \mathscr{K}(\Phi(w))$, we have $|(\nabla v(t))(\mathbf{x})| \leq \Phi(w)$ a.e. on $t \in \mathrm{I}$, $\mathbf{x} \in \Omega$. As $\Phi(w)$ is constant in time, we have $P_{n} v \in \mathscr{K}_{n}(\Phi(w))$. Hence $v_{n}:=\eta_{n} P_{n} v$ belongs to $\mathscr{K}_{n}\left(\Phi_{n}\left(P_{n} w_{n}\right)\right)$, i.e., B5(ii) holds.

Consider now B5(ii) in the obstacle-type case. As before, we have $\Gamma_{n}\left(P_{n} w_{n}\right)=$ $\Gamma\left(E_{n} P_{n} w_{n}\right) \rightarrow \Gamma(w)$. Hence $\Gamma\left(E_{n} P_{n} w_{n}\right) \phi_{i} \rightarrow \Gamma(w) \phi_{i}$ in $L^{\infty}(\Omega)$ for $i=1,2$. Since $\Gamma\left(E_{n} P_{n} w_{n}\right) \phi_{1} \leq 0 \leq \nu \leq \Gamma\left(E_{n} P_{n} w_{n}\right) \phi_{2}$, similarly with the paragraph above, there exists $\left\{\eta_{n}\right\}$ such $\eta_{n} \uparrow 1$ with

$$
\Gamma\left(E_{n} P_{n} w_{n}\right) \phi_{1} \leq \eta_{n} \Gamma(w) \phi_{1} \leq 0 \leq \eta_{n} \Gamma(w) \phi_{2} \leq \Gamma\left(E_{n} P_{n} w_{n}\right) \phi_{2} .
$$

Again, as $v \in \mathscr{K}(\Phi(w))$, we have $P_{n} v \in \mathscr{K}_{n}(\Phi(w))$, and $v_{n}:=\eta_{n} P_{n} v$ belongs to $\mathscr{K}_{n}\left(\Phi_{n}\left(P_{n} w_{n}\right)\right)$.

We are now in the position to state how the solution mappings of $(\mathrm{P})$ and $\left(\sqrt{\mathrm{P}^{n}}\right)$ are related by means of the weak topology on $\mathscr{V}$.

TheOrem 4.5. Given $w \in \mathscr{V}$, let $u=T(w) \in \mathscr{D}(L ; \mathscr{V}) \cap \mathscr{K}(\Phi(w))$, where $T(w)$ is defined as the solution to

$$
(L u+\mathcal{A}(u)-f, v-u) \geq 0, \quad \forall v \in \mathscr{K}(\Phi(w)),
$$

and, similarly, $u_{n}=T_{n}(z) \in \mathscr{K}_{n}\left(\Phi_{n}(z)\right)$, with $z \in \mathscr{V}_{n}$, where $T_{n}(z)$ denotes the solution to

$$
\left(L_{n} u_{n}+\mathcal{A}\left(u_{n}\right)-f_{n}, v-u_{n}\right)_{\mathscr{V}_{n}^{\prime}, \mathscr{V}_{n}} \geq 0, \quad \forall v \in \mathscr{K}_{n}\left(\Phi_{n}(z)\right)
$$


Then, if $w_{n} \rightarrow w$ in $\mathscr{V}$,

$$
E_{n} T_{n}\left(P_{n} w_{n}\right) \rightarrow T(w) \text { in } \mathscr{V}
$$

Proof. Both maps $T: \mathscr{V} \rightarrow \mathscr{V}$ and $T_{n}: \mathscr{V}_{n} \rightarrow \mathscr{V}_{n}$ are well-defined and single valued since $\mathscr{K}(\Phi(w))$ and $\mathscr{K}_{n}\left(\Phi_{n}\left(P_{n} w_{n}\right)\right)$ are closed, convex (in $\mathscr{V}$ and $\mathscr{V}_{n}$, respectively) and contain 0 , respectively.

By definition, $u_{n}:=T_{n}\left(P_{n} w_{n}\right) \in \mathscr{K}_{n}\left(\Phi_{n}\left(P_{n} w_{n}\right)\right)$ and the usual monotonicity trick gives $\left|u_{n}\right|_{\mathscr{V}_{n}} \leq\left(\left|f_{n}\right|_{\mathscr{V}_{n}^{\prime}} / c\right)^{1 /(r-1)}$. By assumption B2 we have $f_{n}=P_{n} f$, and, thus, the sequence $\left\{\left|f_{n}\right|\right\}_{\mathscr{V}_{n}^{\prime}}$ is uniformly bounded. Indeed, it holds that $\left|f_{n}\right|_{\mathscr{V}_{n}^{\prime}}=$ $\left|f_{n}\right|_{\mathscr{V}_{n}} \leq\left|P_{n}\right|_{\mathscr{L}\left(\mathscr{V}, \mathscr{V}_{n}\right)}|f|_{\mathscr{V}} \leq|f|_{\mathscr{V}}$. Then the sequence $\left\{\left|u_{n}\right|_{\mathscr{V}_{n}}\right\}$ is bounded uniformly, as well. Since we have the uniform bound $\left|E_{n}\right|_{\mathscr{L}\left(\mathscr{V}_{n}, \mathscr{V}\right)} \leq 1$, the sequence $\left\{E_{n} u_{n}\right\}$ is uniformly bounded in $\mathscr{V}$. By the reflexivity of $\mathscr{V}$, there exists a weakly convergent subsequence, i.e., $E_{n} u_{n} \rightarrow u$ in $\mathscr{V}$ for $n \in \mathbb{N}^{\prime} \subset \mathbb{N}$. This and B5(i) now imply that $u \in \mathscr{K}(\Phi(w))$.

Next, define $\tilde{\mathcal{A}}_{n}(\cdot):=P_{n}^{\prime} \mathcal{A}\left(P_{n} \cdot\right)$ and $\tilde{f}_{n}=P_{n}^{\prime} f_{n}$, where $P_{n}^{\prime}: \mathscr{V}_{n}^{\prime} \rightarrow \mathscr{V}^{\prime}$. Minty's Lemma yields that (4.8) holds when " $L u_{n}$ " is exchanged by " $L v$ " with $v \in \mathscr{K}_{n}\left(\Phi_{n}\left(P_{n} w_{n}\right)\right)$. Since $P_{n} E_{n}=I=\mathrm{id}$ in $\mathscr{V}_{n}$ for all $n \in \mathbb{N}$, 4.8 implies that

$$
\left(P_{n}^{\prime} L_{n} v+\tilde{\mathcal{A}}\left(E_{n} u_{n}\right)-\tilde{f}_{n}, E_{n} v-E_{n} u_{n}\right) \geq 0, \quad \forall v \in \mathscr{K}_{n}\left(\Phi_{n}\left(P_{n} w_{n}\right)\right) .
$$

Let $v \in \mathscr{D}\left(L ; \mathscr{V}^{\prime}\right) \cap \mathscr{K}(\Phi(w))$, then by B5(ii) there exists a real-valued sequence $\left\{\eta_{n}\right\}$ such $\lim _{n \rightarrow \infty} \eta_{n}=1$ for which $\eta_{n} P_{n} v \in \mathscr{K}_{n}\left(\Phi_{n}\left(P_{n} w_{n}\right)\right)$. Define $v_{n}=\eta_{n} P_{n} v$. Since $E_{n} P_{n}$ converges strongly to the identity (A2, Assumption 1) and $\eta_{n} \rightarrow 1$, we have $E_{n} v_{n} \rightarrow v$ in $\mathscr{V}$ as $n \rightarrow \infty$. Using $v=v_{n}$ in 4.9, we obtain

$$
\left(\tilde{\mathcal{A}}_{n}\left(E_{n} u_{n}\right), E_{n} u_{n}-u\right) \leq\left(\eta_{n} P_{n}^{\prime} L_{n} P_{n} v-\tilde{f}_{n}, E_{n} v_{n}-E_{n} u_{n}\right)+\left(\tilde{\mathcal{A}}_{n}\left(E_{n} u_{n}\right), E_{n} v_{n}-u\right) .
$$

From the first paragraph of the proof, we have that $\left\{E_{n} u_{n}\right\}$ is bounded in $\mathscr{V}$. Since $\mathcal{A}$ maps bounded sets into bounded sets and the norms of $P_{n}$ and $P_{n}^{\prime}$ are uniformly bounded in $n \in \mathbb{N}\left(\left|P_{n}\right|_{\mathscr{L}\left(\mathscr{V}, \mathscr{V}_{n}\right)} \leq 1\right.$ and hence also $\left.\left|P_{n}^{\prime}\right|_{\mathscr{L}\left(\mathscr{V}_{n}^{\prime}, \mathscr{V}^{\prime}\right)} \leq 1\right)$, we have that $\left\{\tilde{\mathcal{A}}_{n}\left(E_{n} u_{n}\right)\right\}$ is bounded. By the reflexivity of $\mathscr{V}^{\prime}$, there exists a subsequence converging weakly to some $g \in \mathscr{V}^{\prime}$. Also, we have that $P_{n}^{\prime} L_{n} P_{n} v \rightarrow L v$ since $v \in$ $\mathscr{D}\left(L ; \mathscr{V}^{\prime}\right)$ (see Proposition 1 in [17]) and hence $\eta_{n} P_{n}^{\prime} L_{n} P_{n} v \rightarrow L v$ as $n \rightarrow \infty$. By our hypotheses, we further have $\tilde{f}_{n}=P_{n}^{\prime} f_{n}=P_{n}^{\prime} P_{n} f=E_{n} P_{n} f \rightarrow f$ in $\mathscr{V}^{\prime}$ (actually in $\mathscr{V})$ as $n \rightarrow \infty$. Summarising, we have the following relations:

$\tilde{\mathcal{A}}_{n}\left(E_{n} u_{n}\right) \rightarrow g, \tilde{f}_{n} \rightarrow f, \eta_{n} P_{n}^{\prime} L_{n} P_{n} v \rightarrow L v$ in $\mathscr{V}^{\prime} \quad$ and $\quad E_{n} u_{n} \rightarrow u, E_{n} v_{n} \rightarrow v$ in $\mathscr{V}$.

Henceforth, taking "im" in 4.10, we obtain

$$
\varlimsup_{n \rightarrow \infty}\left(\tilde{\mathcal{A}}_{n}\left(E_{n} u_{n}\right), E_{n} u_{n}-u\right) \leq(L v-f+g, v-u) .
$$

Let $v=v_{\alpha} \in \mathscr{D}\left(L ; \mathscr{V}^{\prime}\right) \cap \mathscr{K}(\Phi(w))$ with $\lim _{\alpha \rightarrow 0} v_{\alpha}=u$ and $\left(L v_{\alpha}, v_{\alpha}-u\right) \leq 0$ (which is possible due to the compatibility of $S(\tau)$ and $\mathscr{K}(\Phi(w))$, see [25, 7]). This choice implies $\varlimsup_{n \rightarrow \infty}\left(\tilde{\mathcal{A}}_{n}\left(E_{n} u_{n}\right), E_{n} u_{n}-u\right) \leq 0$. Here we also have assumed that $\mathcal{A}$ is the time realization of a linear uniformly monotone operator $A$ in $V$ (B3 of Assumption 2), i.e., $\mathcal{A}(y)(t)=A(y(t))$ for $t \in \mathrm{I}$ and $y \in \mathscr{V}=L^{p}(\mathrm{I} ; V)$. Consequently, we have 
$P_{n}^{\prime} \mathcal{A}\left(P_{n} v\right)=P_{n}^{\prime} A\left(P_{n} v(t)\right)$ and hence

$$
\begin{aligned}
\left(P_{n}^{\prime} \mathcal{A}\left(P_{n} y\right), z\right) & =\int_{0}^{T} \sum_{m=1}^{n}\left(A\left(\frac{1}{h} \int_{\mathrm{I}_{m}} y(t) \mathrm{d} t\right), z(t)\right)_{V^{\prime}, V} \chi_{\mathrm{I}_{m}}(t) \mathrm{d} t= \\
& =\int_{0}^{T}\left(A\left(\sum_{m=1}^{n} \frac{1}{h} \int_{\mathrm{I}_{m}} y(t) \mathrm{d} t \chi_{\mathrm{I}_{m}}(t)\right), z(t)\right)_{V^{\prime}, V} \mathrm{~d} t= \\
& =\left(\mathcal{A}\left(E_{n} P_{n} y\right), z\right) .
\end{aligned}
$$

The relation $P_{n} E_{n}=I$ thus yields $P_{n}^{\prime} \mathcal{A}\left(P_{n}\left(E_{n} u_{n}\right)\right)=\mathcal{A}\left(E_{n} u_{n}\right)$. This and 4.11) imply

$$
\varlimsup_{n \rightarrow \infty}\left(\mathcal{A}\left(E_{n} u_{n}\right), E_{n} u_{n}-u\right) \leq 0 .
$$

Since the operator $\mathcal{A}$ is pseudomonotone (it satisfies $\mathbf{H 1}$ and $\mathbf{H} \mathbf{3}$ which imply pseudomonotinicity, see the paragraph that follows the definition of H1-H3 and see [37] for a proof) and $\tilde{\mathcal{A}}_{n}\left(E_{n} u_{n}\right)=P_{n}^{\prime} \mathcal{A}\left(P_{n}\left(E_{n} u_{n}\right)\right)=\mathcal{A}\left(E_{n} u_{n}\right)$, we observe

$$
(\mathcal{A}(u), u-z) \leq \varliminf_{n \rightarrow \infty}\left(\mathcal{A}\left(E_{n} u_{n}\right), E_{n} u_{n}-z\right)=\varliminf_{n \rightarrow \infty}\left(\tilde{\mathcal{A}}_{n}\left(E_{n} u_{n}\right), E_{n} u_{n}-z_{n}\right),
$$

for all $z \in \mathscr{V}$ and $\left\{z_{n}\right\}$ such $z_{n} \rightarrow z$ in $\mathscr{V}$.

Let $z \in \mathscr{D}\left(L ; \mathscr{V}^{\prime}\right) \cap \mathscr{K}(\Phi(w))$ be arbitrary. Then by B5(ii) there exists a real-valued sequence $\left\{\eta_{n}\right\}$ with $\lim _{n \rightarrow \infty} \eta_{n}=1$. We next define $z_{n}:=\eta_{n} P_{n} z \in$ $\mathscr{K}_{n}\left(\Phi_{n}\left(P_{n} w_{n}\right)\right)$ and have $E_{n} z_{n} \rightarrow z$ in $\mathscr{V}$. Assigning $v=z_{n}$ in 4.9 , we observe that

$$
\varliminf_{n \rightarrow \infty}\left(\tilde{\mathcal{A}}_{n}\left(E_{n} u_{n}\right), E_{n} u_{n}-E_{n} z_{n}\right) \leq(f-L z, u-z) .
$$

The above, together with 4.13), yields that $u \in \mathscr{K}(\Phi(w))$ satisfies

$$
(L z+\mathcal{A}(u)-f, z-u) \geq 0, \text { for all } z \in \mathscr{D}\left(L ; \mathscr{V}^{\prime}\right) \cap \mathscr{K}(\Phi(w)),
$$

i.e., $u$ solves $(\mathrm{wP}$. The increased regularity of $f$, the uniform monotonicity of $A$ and the $S(\tau)$-invariance of $\mathscr{K}(\Phi(w))$ yield $u \in \mathscr{D}(L ; \mathscr{V})$ (see [25, 7]). Hence $u$ also solves (P).

Finally, from 4.12, the uniform monotonicity of $\mathcal{A}$ and the fact that $E_{n} u_{n} \rightarrow u$, we have

$$
c \varlimsup_{n \rightarrow \infty}\left|E_{n} u_{n}-u\right|_{\mathscr{V}}^{r} \leq \varlimsup_{n \rightarrow \infty}\left(\mathcal{A}\left(E_{n} u_{n}\right)-\mathcal{A}(u), E_{n} u_{n}-u\right) \leq 0
$$

i.e., $E_{n} u_{n} \rightarrow u$ in $\mathscr{V}$, along a subsequence.

Suppose that there exists a subsequence of $\left\{u_{n}\right\}:=\left\{T_{n}\left(P_{n} w_{n}\right)\right\}$ which does not converge to the solution $u$ determined above. Hence, there is $\epsilon>0$ such that $\left|u_{n_{i}}-u\right| \geq \epsilon$ for $i \in \mathbb{N}$. On the other hand, we can apply the same reasoning as above to $\left\{u_{n_{i}}\right\}$ which yields the existence of a subsequence of $\left\{u_{n_{i}}\right\}$ converging to $u^{*}$ that solves Problem $(\mathrm{P})$. Theorem 3.2 and Corollary 3.3 establish uniqueness of the solution, which implies that $u^{*}=u$. Thus, the entire sequence $\left\{u_{n}\right\}$ satisfies $E_{n} u_{n} \rightarrow u$. 
Finally we state the result required for the numerical approximation of the parabolic QVI of interest.

Corollary 4.6. Let $f_{n}=P_{n} f=\left\{f^{m}\right\}_{m=1}^{n}$, and let, for each $n \in \mathbb{N}, u_{n}=$ $\left\{u^{m}\right\}_{m=1}^{n} \in \mathscr{V}_{n}$ be the unique solution to

$$
\begin{aligned}
& \qquad \begin{array}{l}
u^{m} \in \mathbf{K}\left(\Phi_{n}\left(u_{n}\right)\right): \\
\left(\frac{u^{m}-u^{m-1}}{h}+A\left(u^{m}\right)-f^{m}, v^{m}-u^{m}\right)_{V} \geq 0, \forall v^{m} \in \mathbf{K}\left(\Phi_{n}\left(u_{n}\right)\right) ; \quad\left(\mathrm{N}_{\mathrm{QVI}}\right) \\
u^{1}=0, \\
\text { for } m=2, \ldots, n \text { and with } h=T / n \text {. Then, } \\
\qquad E_{n} u_{n} \rightarrow u^{*}, \text { in } \mathscr{V} \text { as } n \rightarrow \infty,
\end{array}
\end{aligned}
$$

where $u^{*}$ solves $(\mathrm{P})$.

Proof. Combining Theorem 4.1 and Theorem 4.5 proves the assertion. $\square$

4.1. Solution Algorithm. The previous results yield Algorithm 1 below for computing the solution to $\left(\mathrm{N}_{\mathrm{QVI}}\right.$. In its statement the term "Suitable Convergence Criteria" refers to a stopping rule associated with the fixed point equation $u=S_{n}\left(\mathcal{A}, f_{n}, \mathscr{K}_{n}\left(\Phi_{n}(u)\right)\right)$. In our case, and following [17, we use a criterion based on the linear convergence of the approximate sequence defined in Step 3 of Algorithm 1. see $\left(\mathrm{SP}_{\text {conv. }}\right)$ below.

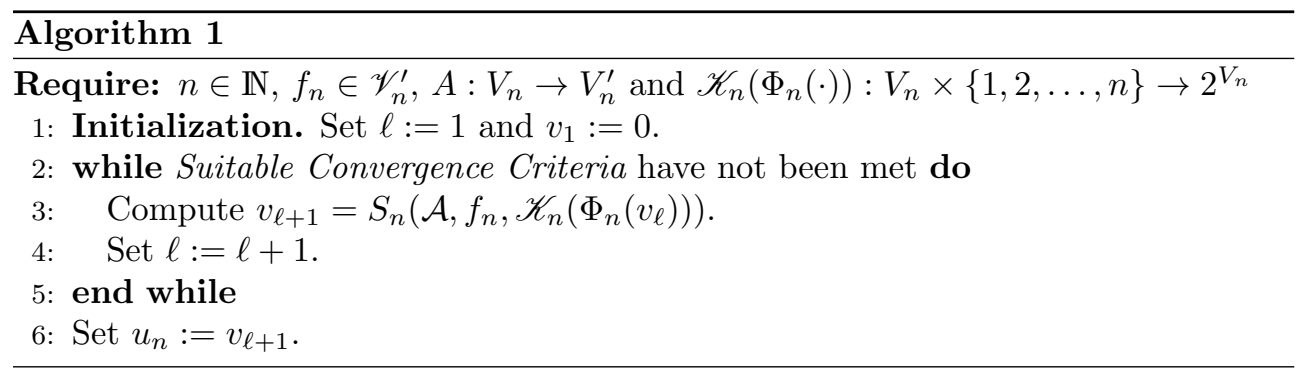

5. Numerics. In this section we are concerned with computing an approximate solution to $(\mathrm{P})$ by means of solving the approximating problem $\left(\mathrm{N}_{\mathrm{QVI}}\right)$, where the operator $\mathcal{A}$ is the time realization of the $p$-Laplacian, with $p=2$ or $p=3$. We use $\mathrm{I}=(0,1)$ and $\Omega=(0,1) \times(0,1)$ in all examples below. The state space is given by $\mathscr{V}=L^{p}(\mathrm{I} ; V)$ with $V=W_{0}^{1, p}(\Omega)$ with the Gelfand triple structures $\left(\mathscr{V}, \mathscr{H}, \mathscr{V}^{\prime}\right)$ and $\left(V, H, V^{\prime}\right)$ with $\mathscr{H}=L^{2}(\mathrm{I} ; H)$ and $H=L^{2}(\Omega)$. All our test examples are of gradient-type.

The discretization in time is realised by considering $\left(\overline{\mathrm{N}_{\mathrm{QVI}}}\right)$ where the uniform mesh size is given by $h=T / n$ on $\mathrm{I}=(0,1)$. Our finite difference approximation scheme in space has $M^{2}$ uniformly distributed nodes implying the mesh size $k=$ $1 /(M+1)$ in each coordinate direction. At a node $\mathbf{x}_{i j}=\left(x_{i}, x_{j}\right)$, with $x_{i}=i k$ and $x_{j}=j k$ for $1 \leq i, j \leq M$, we approximate $w\left(\mathbf{x}_{i j}\right)$, for $w \in V$, by $w_{i j}=w\left(x_{i}, x_{j}\right)$ and denote by $w^{k}$ the corresponding discrete approximation of $w$ on the given mesh. We approximate the $V$-norm by $\left|w^{k}\right|_{V}^{p}:=\sum_{i, j=1}^{M}\left|\left(D_{-} w^{k}\right)\right|_{i j}^{p} k^{2}$ with $\left(D_{-} w^{k}\right)_{i j}=$ $\frac{1}{k}\left(w_{i j}-w_{(i-1) j}, w_{i j}-w_{i(j-1)}\right)^{\top}$ and $\left|\left(u^{k}, v^{k}\right)^{\top}\right|_{i j}^{2}=u_{i j}^{2}+v_{i j}^{2}$. The approximation of the $\mathscr{V}$-norm is given by $|v|_{\mathscr{V}_{n}}^{p}=\sum_{j=1}^{n} h\left|v_{j}^{k}\right|_{V}^{p}$ with $v=\sum_{j} v_{j}^{k} \chi_{\mathrm{I}_{j}}$. The discretization of 
the second order elliptic operator $-\Delta_{p}: W_{0}^{1, p}(\Omega) \rightarrow W^{-1, p^{\prime}}(\Omega)$ is based on a second order accurate five-point centered difference scheme. More details on this scheme can be found in [19, 16].

In all the examples, we have $f(t)=g(t) \psi$, where $g \in C^{1}(\overline{\mathrm{I}})$ with $g(0)=1$ and $\psi \in W_{0}^{1, p}(\Omega) \cap C^{\infty}(\Omega)$. In particular, we choose $\psi(x, y)=N(x y(x-1)(y-1))^{2}$ with $N$ a normalization constant such that $\psi(1 / 2,1 / 2)=1$. The forcing term is then given by

$$
f(t, x, y)=r_{1}\left(1-e^{-r_{3} t^{r_{2}}}\right) \psi(x, y),
$$

where $r_{1}, r_{2}, r_{3}>0$ are chosen differently for each example.

For the sequence $\left\{v_{\ell}\right\}$ generated as in Step 3 of Algorithm 1 we define the linear convergence coefficient sequence $\left\{\mu^{\ell}\right\}$ by $\mu^{\ell}:=\left|v_{\ell+2}-v_{\ell+1}\right|_{\mathscr{V}_{n}} /\left|v_{\ell+1}-v_{\ell}\right|_{\mathscr{V}_{n}}$. The convergence criteria of Algorithm 1 are considered satisfied as soon as for some $\ell>\ell_{0}$

$$
\left.\begin{array}{l}
\max _{\ell-\ell_{0} \leq r, s \leq \ell}\left|\mu^{r}-\mu^{s}\right|<\epsilon_{1}, \\
\frac{\left|v_{2}-v_{1}\right|_{\mathscr{\vartheta}}}{1-\mu^{\ell}} \prod_{i=1}^{\ell} \mu^{i}<\epsilon_{2},
\end{array}\right\}
$$

with some prescribed $\ell_{0} \in \mathbb{N}, \epsilon_{1}>0$ and $\epsilon_{2}>0$. Then, Algorithm11 is stopped. In our numerical tests, using $\ell_{0}=4, \epsilon_{1}=1 \mathrm{e}-2$ and $\epsilon_{2}=1 \mathrm{e}-4$, the conditions in $\mathrm{SP}_{\text {conv. }}$. are satisfied for $\ell=8$ in Examples 1 and 2, and $\ell=14$ for Example 3. For a detailed explanation of these convergence criteria we refer to [16]. The values of the linear convergence coefficients $\left\{\mu^{\ell}\right\}$ satisfy $\mu^{\ell} \leq 0.15$ in the first example and $\mu^{\ell} \leq 0.13$ in the second one for $\ell \leq 8$. The behavior of these coefficients is stable under mesh refinements for $h=2^{-n}$ for $n=5,6,7$ (i.e., there are no substantial differences on the bounds for $\left\{\mu^{\ell}\right\}$ under mesh refinements). Although Example 3 does not fall into the scope of Theorem 3.2 (the $p$-Laplacian for $p=3$ does not satisfies the necessary hypothesis for the theorem to hold) the algorithm nevertheless exhibits linear convergence. On the other hand, this behavior appears unstable under perturbations of the forcing term. In fact (slight) variations of $f$ (for example considering Example 3 with the forcing term of Examples 2 and 1) make the algorithm non-convergent. This is substantially different for elliptic QVIs; compare [16].

The computation in Step 3 of Algorithm 1 is based on a penalty-method combined with a semismooth Newton iteration. This approach was successfully applied in [16] and [17] and the reader is referred to these references for further details. In our examples, we stop the Newton iteration when the norm of the distance between two successive iterates is below NewtonTol $=1 \mathrm{e}-5$. The total number of iterations for the semismooth Newton algorithm, using the continuation technique for the penalty parameter described in [16, remained stable under mesh refinements. The behavior in each time step is analogous to the one reported in [16].

The computational domain consists of $M^{2}$ uniformly distributed nodes in $\Omega=$ $(0,1) \times(0,1)$, where $M=128$ and the mesh size is $k=1 /(\mathrm{M}+1))$. The time interval $\mathrm{I}=(0,1)$ is discretized uniformly with mesh size $h=1 / 100$

5.1. Example 1. Let $\mathcal{A}=-\Delta$, with $r_{1}=0.1, r_{2}=2$ and $r_{3}=10$ and with $\Phi(v)(t)$ determined by

$$
\Phi(v)=\left(\left|\int_{0}^{1}\left(\int_{\Omega} v(s, \mathbf{x}) \mathrm{d} \mathbf{x}\right) \mathrm{d} s\right|+0.001\right)(0.2+0.8 \psi(x, y))
$$


The forcing term $t \mapsto f(t)$ at $t=0.01,0.12,1$ is shown in Figures 5.1(a), 5.1(b) and 5.1(c) and the approximate solution, $t \mapsto u(t)$, to the QVI is depicted at the same time steps in Figures 5.1(d), 5.1(e) and 5.1(f), The behavior of the norm of the gradient $t \mapsto|\nabla u(t)|$ is shown in Figures $5.1(\mathrm{~g})$, 5.1(h) and 5.1(i), also at the same time steps, and finally the approximation of the active set $t \mapsto \mathbb{A}(t)=\{\mathbf{x} \in \Omega$ : $|\nabla u(t, \mathbf{x})|-\Phi(u)(\mathbf{x})=0\}$ at times $t=0.12,1$ is depicted in Figures 5.1(j) and 5.1(k)

The spatial part of the gradient bound $\Phi(v)$ is proportional to $(0.2+0.8 \psi)$ with the latter being a concave function with maximum in the center of the square and minimum on the sides of the square. We also note that the finite difference scheme is an implicit one. Therefore whenever the solution at a time step is inactive in $\Omega$, it is the solution of an elliptic problem where the second order operator is the Laplacian. Since solutions of such problems satisfy maximum principles for the gradient (i.e. the supremum of the norm of the gradient is obtained at the boundary) it is expected that the solution hits activity starting from regions on the sides of the square (this is observed in Figure 5.1(j) . On the other hand, $f$ forces the norm of the gradient of the solution to keep growing (in the inactive parts) as time evolves such that the solution at $t=1$ has a large active set as can be seen in Figure 5.1(k). Finally, due to the constraint, the maximum of the norm of the gradient is no longer found on the boundaries as it would be expected in the unconstrained version of the problem.

5.2. Example 2. Let $\mathcal{A}=-\Delta$, with $r_{1}=0.1, r_{2}=2$ and $r_{3}=10$ and with $\Phi(v)(t)$ determined by

$$
\Phi(v)(t)=\left(\left|\int_{0}^{1}\left(\int_{\Omega} v(s, \mathbf{x}) \mathrm{d} \mathbf{x}\right) \mathrm{d} s\right|+0.001\right)(1-0.2 \psi(x, y)) .
$$

The forcing term $t \mapsto f(t)$ is the same as in Example 1. The approximated solution, $t \mapsto u(t)$, to the QVI at the time steps $t=0.01,0.12,1$ is shown in Figures $5.2(\mathrm{a})$ 5.2(b) and 5.2(c). The behavior of the norm of the gradient $t \mapsto|\nabla u(t)|$ is displayed in Figures $5.2(\mathrm{~d}), 5.2(\mathrm{e})$ and $5.2(\mathrm{f})$, also at the same time steps. Finally, the approximation of the active set $t \mapsto \mathbb{A}(t)=\{\mathbf{x} \in \Omega:|\nabla u(t, \mathbf{x})|-\Phi(u)(\mathbf{x})=0\}$ at times $t=0.12,1$ can be observed in Figures $5.2(\mathrm{~g})$ and $5.2(\mathrm{~h})$.

In this example, the spatial part of the gradient bound $\Phi(v)$ is proportional to $(1-0.2 \psi)$, which is a convex function with a minimum in the center of the square and maximum on the boundary of the square. As discussed in the previous example, in each time step without activity, the maximum of the norm of the gradient is expected at the boundaries. However, given the convexity of the constraint, the approximate solution to the QVI hits activity in a region inside the domain as can be seen in Figures $5.2(\mathrm{~g})$,

5.3. Example 3. Let $\mathcal{A}=-\Delta_{p}$, with $p=3$, with $r_{1}=0.01, r_{2}=2$ and $r_{3}=0.15$ and with $\Phi(v)(t)$ determined by

$$
\Phi(v)(t)=\left(\left|\int_{0}^{1}\left(\int_{\Omega} v(s, \mathbf{x}) \mathrm{d} \mathbf{x}\right) \mathrm{d} s\right|+0.001\right)
$$

The forcing term $t \mapsto f(t)$ at $t=0.01,0.12,1$ is shown in Figures 5.3(a), 5.3(b) and 5.3(c), and the approximate solution, $t \mapsto u(t)$, to the QVI is depicted at the same time steps in Figures 5.3(d), 5.3(e) and 5.3(f) The behavior of the norm of the gradient $t \mapsto|\nabla u(t)|$ is shown in Figures 5.3(g), 5.3(h) and 5.3(i), also at the same time steps, and finally the approximation of the active set $t \mapsto \mathbb{A}(t)=\{\mathbf{x} \in \Omega$ : 


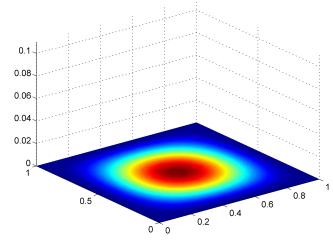

(a)

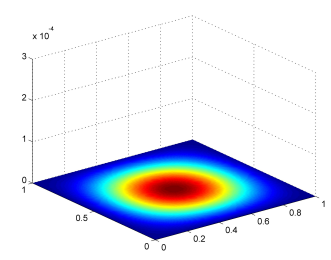

(d)

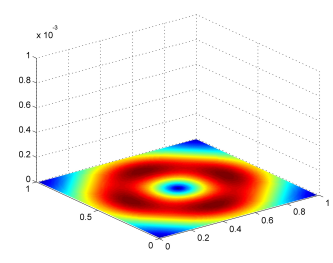

(g)

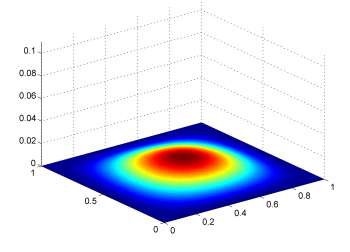

(b)

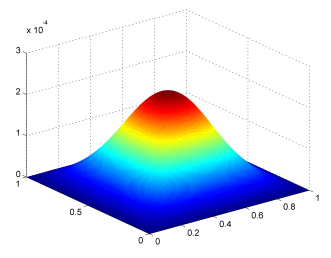

(e)

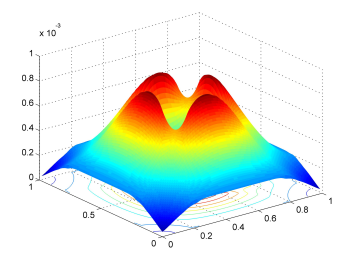

(h)

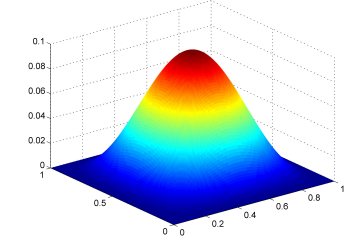

(c)

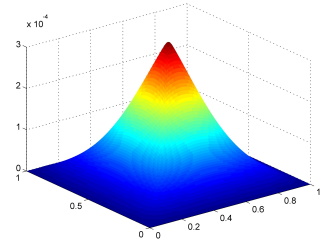

(f)

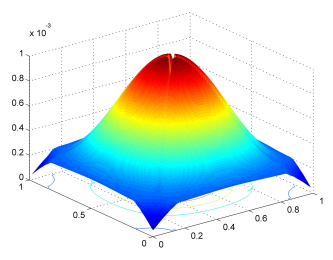

(i)

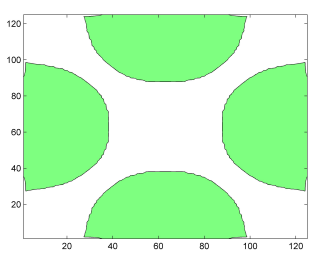

(j)

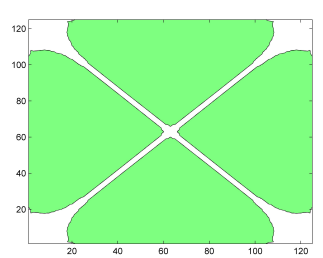

(k)

FIG. 5.1. $\mathbf{x} \mapsto f(t, \mathbf{x})$ for $t=0.01, t=0.12$ and $t=1$ in $5.1(a)$ 5.1(b) and 5.1(c) respectively. $\mathbf{x} \mapsto u(t, \mathbf{x})$ for $t=0.01, t=0.12$ and $t=1$ in $5.1(d), 5.1(e)$ and $5.1(f)$, respectively. $\mathbf{x} \mapsto|\nabla u(t, \mathbf{x})|$ for $t=0.01, t=0.12$ and $t=1 \mathrm{in} 5.1(\mathrm{~g})$, $5.1(\mathrm{~h})$ and $5.1(\mathrm{i})$, respectively. 5.1(j) Active set at time $t=0.12$. 5.1(k) Active set at time $t=1$

$|\nabla u(t, \mathbf{x})|-\Phi(u)(\mathbf{x})=0\}$ at times $t=0.89,0.94$ can be observed in Figures 5.3(j) and $5.3(\mathrm{k})$

6. Discussion and Further Research. In Theorem 3.2 a contraction result for the mapping $v \mapsto S(\mathcal{A}, f, \mathscr{K}(\Phi(v)))$ is provided, when $\Phi(v)=\Gamma(v) \phi$ for some $\phi$ and $\Gamma$ a Lipschitz continuous functional. Given the structure of the proof of the aforementioned theorem, it is not trivial to extend the result to operators of higher rank, as for example when $\Phi(v)=\sum_{i}^{n} \Gamma_{i}(v) \phi_{i}$. Another open question is wether the class of operators $\mathcal{A}$, under which a contractive behavior is observed, can be extended to operators such as the $p$-Laplacian. Theorem 3.2 is an extension of a result in [16] 


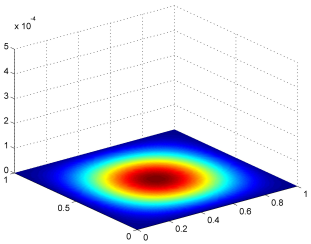

(a)

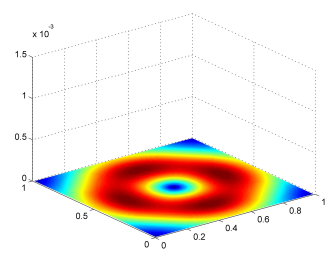

(d)

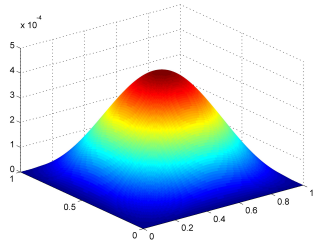

(b)

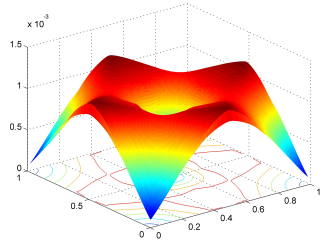

(e)

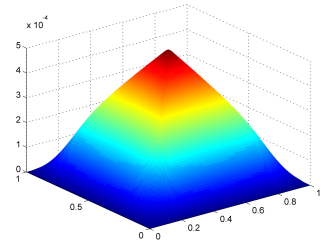

(c)

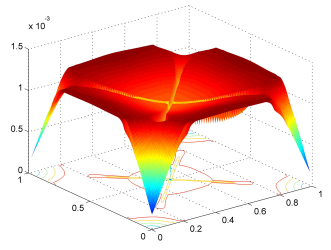

(f)

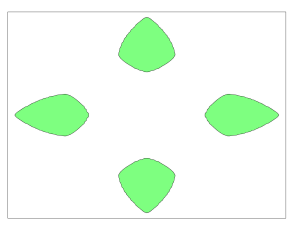

$(\mathrm{g})$

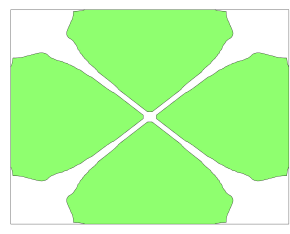

(h)

FIG. 5.2. $\mathbf{x} \mapsto u(t, \mathbf{x})$ for $t=0.01, t=0.15$ and $t=1$ in 5.2(a) 5.2(b) and 5.2(c) respectively. $|\nabla u(t, \mathbf{x})|$ for $t=0.01, t=0.15$ and $t=1$ in 5.2(d), 5.2(e) and 5.2(f) respectively $5.2(\mathrm{~g})$ Active set at time $t=0.15 .5 .2(h)$ Active set at time $t=1$

for elliptic QVIs where several numerical tests show the linear convergence behavior for the $p$-Laplacian case, when $p=3$. Such a good convergence behavior seems much more delicate to obtain in the parabolic case as stated in $\$ 5$.

The structure of the constraint sets $\mathscr{K}(\Phi(v))=\{w \in \mathscr{V}: w(t) \in \mathbf{K}(\Phi(v))$ a.e. $t \in$ I\} under the hypothesis of Theorem 3.2, i.e., with $\Phi(v)=\Gamma(v) \phi$ and $\Gamma$ a nonlinear Lipschitz continuous functional, implies at time $t$ that the information on the bound $\Phi(v)$ of the state variable $u(t)$ comes from the entire interval I. A scheme for causal sets, i.e., when the solution to the QVI at time $t, u(t)$, can be obtained as a solution to a QVI where the constraint set depends only on the set $\{v: v=u(\tau)$ for $0 \leq \tau \leq t\}$ was developed on [17. However, it is not known under what conditions on these types of constraints solutions are unique. An answer to this question is of paramount importance.

The axiomatic approximation scheme developed in 84 appears to be suitable to be extended to a fully discretized scheme. For parabolic VIs, such a path was followed by Glowinski, Lions and Trémolières in [12. However, in the QVI case the discretization of the constraint set mapping $v \mapsto \mathscr{K}(\Phi(v))$ requires special attention, and conditions for this discretization to be useful for approximation methods are currently unkwown. 


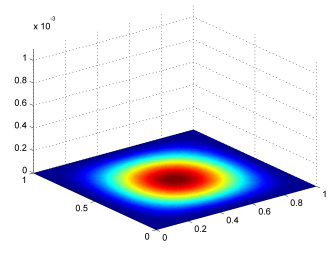

(a)

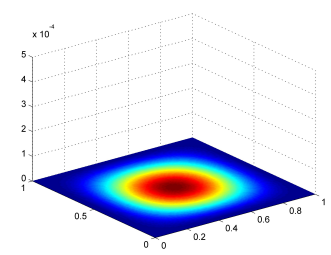

(d)

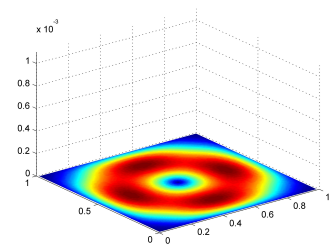

(g)

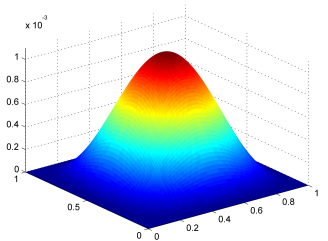

(b)

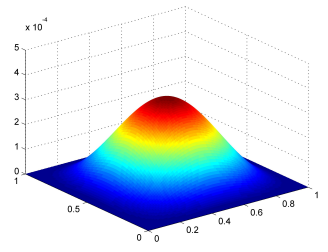

(e)

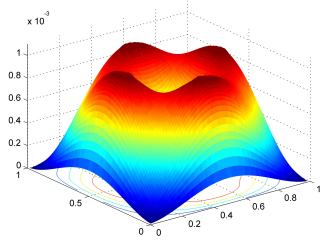

(h)

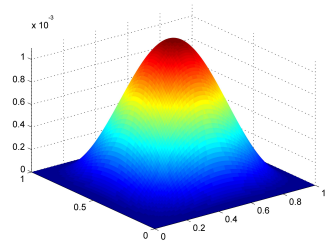

(c)

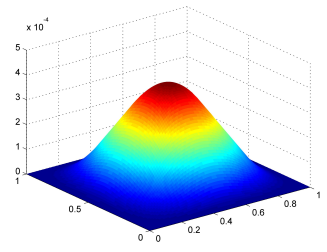

(f)

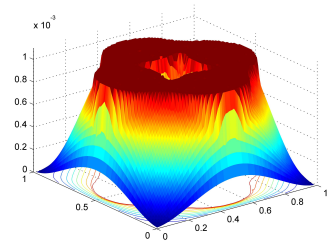

(i)

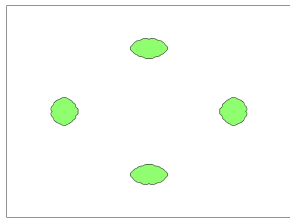

(j)

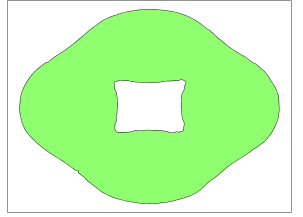

(k)

FIG. 5.3. $\mathbf{x} \mapsto f(t, \mathbf{x})$ for $t=0.01, t=0.89$ and $t=0.94$ in 5.3(a), 5.3(b) and 5.3(c), respectively. $\mathbf{x} \mapsto u(t, \mathbf{x})$ for $t=0.01, t=0.89$ and $t=0.94$ in $5.3(d), 5.3(e)$ and $5.3(f)$ respectively. $|\nabla u(t, \mathbf{x})|$ for $t=0.01, t=0.89$ and $t=0.94$ in $5.3(\mathrm{~g}), 5.3(\mathrm{~h})$ and $5.3(\mathrm{i})$ respectively. $5.3(\mathrm{j})$ Active set at time $t=0.89 .5 .3(k)$ Active set at time $t=0.94$

[1] R. A. Adams and J. J. F. Fournier. Sobolev Spaces. Academic Press, second edition, 2003.

[2] A. Azevedo and L. Santos. Convergence of convex sets with gradient constraint. Journal of Convex Analysis, 11(2):285-301, 2004.

[3] C. Baiocchi and A. Capelo. Variational and Quasivariational Inequalities. Wiley-Interscience, 1984.

[4] A. Bensoussan. Stochastic Control by Functional Analysis Methods. North-Holland, 1982.

[5] A. Bensoussan and J.-L. Lions. Controle impulsionnel et inéquations quasi-variationnelles d'évolutions. C. R. Acad. Sci. Paris, 276:1333-1338, 1974.

[6] P. Beremlijski, J. Haslinger, M. Kocvara, and J. Outrata. Shape optimization in contact problems with Coulomb friction. SIAM J. Optim, 13:561-587, 2002.

[7] H. Brézis. Équations et inéquations non linéaires dans les espaces vectoriels en dualité. Annales de L'Institut Fourier, 18, 1:115-175, 1968. 
[8] Z. Denkowski, S. Migórski, and N. S. Papageorgiou. Introduction to Nonlinear Analysis: Applications. Kluwer, 2003.

[9] G. Duvaut and J.-P. Lions. Les Inéquations en Mécanique et en Physique. Dunod, Paris, 1972.

[10] Francisco Facchinei, Christian Kanzow, and Simone Sagratella. Solving quasi-variational inequalities via their KKT conditions. Math. Program., 144(1-2, Ser. A):369-412, 2014.

[11] G. Fichera. Problema elastotatici con vincoli unilaterali: il problema di signorini con ambigue condizioni al contorno. Mem. Accad. Naz. Lincei, 7(8):91-140, 1964.

[12] R. Glowinski, J.-P. Lions, and R Trémolières. Numerical Analysis of Variational Inequalities. North-Holland, 1981.

[13] B. Hanouzet and J. L. Joly. Convergece uniforme des itérés définissant la solution d'inéquations quasi-variationnelles et application à la régularité. Num. Funct. Anal. and Optimiz., 4:399414, 1979.

[14] P. T. Harker. Generalized Nash games and quasi-variational inequalities. European Journal of Operational Research, 54:81-94, 1991.

[15] E. Hille and R. S. Phillips. Functional Analysis and Semi-groups. American Mathematical Society, 1957.

[16] M. Hintermüller and C. N. Rautenberg. A sequential minimization technique for elliptic quasivariational inequalities with gradient constraints. SIAM J. Optim., 22(4):1224-1257, 2012.

[17] Michael Hintermüller and Carlos N. Rautenberg. Parabolic quasi-variational inequalities with gradient-type constraints. SIAM J. Optim., 23(4):2090-2123, 2013.

[18] R. Kano, Y. Murase, and N. Kenmochi. Nonlinear evolution equations generated by subdifferentials with nonlocal constraints. In Nonlocal and abstract parabolic equations and their applications, volume 86 of Banach Center Publ., pages 175-194. Polish Acad. Sci. Inst. Math., Warsaw, 2009.

[19] C. T. Kelley. Iterative Methods for Linear and Nonlinear Equations. SIAM, 1995.

[20] R. B. Kellogg. Uniqueness in the schauder fixed point theorem. Proceedings of the AMS, 60:207-240, 1976.

[21] N. Kenmochi. Parabolic quasi-variational diffusion problems with gradient constraints. Discrete Contin. Dyn. Syst. Ser. S, 6(2):423-438, 2013.

[22] A. S. Kravchuk and P. J. Neittaanmäki. Variational and Quasi-variational Inequalities in Mechanics. Springer, 2007.

[23] M. Kunze and J.F. Rodrigues. An elliptic quasi-variational inequality with gradient constraints and some of its applications. Mathematical Methods in the Applied Sciences, 23:897-908, 2000.

[24] T. H. Laetsch. A uniqueness theorem for elliptic q. v. i. J. Functional Analysis, 18:286-288, 1975.

[25] J.-L. Lions. Quelques Méthodes de Résolutions des Problémes aux Limites non Linéaires. Dunod, Gauthier-Villars, 1969.

[26] J.-L. Lions. Sur le côntrole optimal des systemes distribuées. Enseigne, 19:125-166, 1973.

[27] J.-L. Lions. Asymptotic behaviour of solutions of variational inequalitites with highly oscillating coefficients. Applications of Methods of Functional Analysis to Problems in Mechanics, Proc. Joint Symp. IUTAM/IMU. Lecture Notes in Mathematics, Springer, Berlin, 503, 1975.

[28] J.-P. Lions and G. Stampacchia. Variational inequalities. Commun. Pure Appl. Math., 20:493$519,1967$.

[29] F. Miranda, J. F. Rodrigues, and L. Santos. A class of stationary nonlinear maxwell systems. Mathematical Models and Methods in Applied Sciences, 19 (10):1883-1905, 2009.

[30] U. Mosco. Convergence of convex setis and solutions of variational inequalities. Advances in Mathematics, 3 (4):510-585, 1969.

[31] J.-S. Pang and M. Fukushima. Quasi-variational inequalities, generalized Nash equilibria, and multi-leader-follower games. Computational Management Science, 3:373-375, 2009.

[32] A. Pazy. Semigroups of Linear Opertors and Applications to Partial Differential Equations. Springer, 1983.

[33] L. Prigozhin. On the Bean critical-state model in superconductivity. European Journal of Applied Mathematics, 7:237-247, 1996.

[34] J. F. Rodrigues. Obstacle Problems in Mathematical Physics. North-Holland, 1987.

[35] J. F. Rodrigues and L. Santos. A parabolic quasi-variational inequality arising in a superconductivity model. Ann. Scuola Norm. Sup. Pisa Cl. Sci., XXIX:153-169, 2000.

[36] L. Santos. Variational problems with non-constant gradient constraints. Portugaliae Mathematica, 59:205-248, 2002.

[37] R. E. Showalter. Monotone Operators in Banach Space and Nonlinear Partial Differential Equations. American Mathematical Society, 1997. 
[38] L. Tartar. Inéquations quasi variationnelles abstraites. C. R. Ac. Sci. Paris, 178:1193-1196, 1974. 